\title{
Heat Exchanger Network Cleaning Scheduling: From Optimal Control to Mixed-Integer Decision Making
}

\author{
Riham Al Ismaili ${ }^{\mathrm{a}}$, Min Woo Lee ${ }^{\mathrm{b}}$, D. Ian Wilson ${ }^{\mathrm{a}}$, Vassilios S. Vassiliadis ${ }^{\mathrm{a}, *}$ \\ ${ }^{a}$ Department of Chemical Engineering and Biotechnology, University of Cambridge, Pembroke Street, \\ Cambridge CB2 3RA, United Kingdom \\ ${ }^{b}$ Department of Chemical Engineering, Keimyung University, 1095 Dalgubeol-daero, Dalseo-gu, Daegu \\ 42601, South Korea
}

\begin{abstract}
An approach for optimising the cleaning schedule in heat exchanger networks (HENs) subject to fouling is presented. This work focuses on HEN applications in crude oil preheat trains located in refineries. Previous approaches have focused on using mixed-integer nonlinear programming (MINLP) methods involving binary decision variables describing when and which unit to clean in a multi-period formulation. This work is based on the discovery that the HEN cleaning scheduling problem is in actuality a multistage optimal control problem (OCP), and further that cleaning actions are the controls which appear linearly in the system equations. The key feature is that these problems exhibit bang-bang behaviour, obviating the need for combinatorial optimisation methods. Several case studies are considered; ranging from a single unit up to 25 units. Results show that the feasible path approach adopted is stable and efficient in comparison to classical methods which sometimes suffer from failure in convergence.

Keywords: Optimal control problem; Bang-bang control; Fouling; Optimisation;

Scheduling; Heat exchanger networks
\end{abstract}

\section{Introduction}

Fouling of heat transfer surfaces is a long-established problem and has been described as "the major unresolved problem in heat transfer" (Taborek et al., 1972). It is one of the most significant issues affecting heat exchanger operation and thus has been depicted as "a nearly universal problem in heat exchanger equipment and design" (Watkinson, 1988). Heat exchanger fouling accounts for $0.25 \%$ of gross national product (GNP) in highly industrialised countries (Pugh et al., 2001).

This major industry-wide problem is caused by the deterioration in heat transfer resulting from fouling and leads to the loss of efficiency in heat exchangers which must be offset. This 
is achieved through process turndown, increased utility consumption with affiliated surge in greenhouse gas emissions until operation requirements such as temperature and pumparound targets are met, or in extreme cases plant shutdown. The reduction of production rates and increased energy consumption lead to economic losses which are more significant in larger networks of heat exchangers that require long continuous operational times between shutdowns, particularly crude distillation unit preheat trains (PHT) on oil refineries (Smaïli et al., 2001).

Based on 1995 figures, the costs associated specifically with crude oil fouling in PHT worldwide were estimated to be of the order of 4.5 billion USD (Pugh et al., 2001). Fouling mitigation techniques include addition of antifoulant chemicals, using more robust heat transfer equipment, and regular cleaning of fouled units. Cleaning of heat exchangers has a negative impact on operating costs due to the unit being taken offline, however with the development of optimisation strategies such as those proposed by Casado (1990), Smaili et al. (1999),Georgiadis and Papageorgiou (2000), Lavaja and Bagajewicz (2004), Ishiyama et al. (2009b), Gonçalves et al. (2014), among others, these costs can be minimised resulting in overall gains due to improved heat transfer of the network over time.

The cleaning scheduling problem is a discrete decision making problem where a decision must be made as to whether cleaning should be performed, and which unit is to be cleaned. It consists of continuous as well as binary decision variables and hence it has combinatorial complexity that is handled traditionally by Branch and Bound (B\&B) methods of one form or another. Due to its combinatorial nature and the existence of nonlinear models, mathematical programming (MP) techniques have been used to solve this mixed integer nonlinear programming (MINLP) problem based on time discretisation (Smaïli et al., 2001). Additionally this problem has been solved by formulating certain models from a MINLP model to a mixed integer linear programming (MILP) model (Georgiadis and Papageorgiou, 2000). Stochastic optimisation frameworks using distinctive modifications of simulated annealing algorithms have been implemented (Smaïli et al., 2002a) as well as heuristic schemes composed 
of a set of movements according to a greedy rationale (Gonçalves et al., 2014).

This problem has been addressed in the literature through extending the formulation of the general cleaning scheduling problem in a multitude of ways. Rodriguez and Smith (2007) combined the conventional cleaning scheduling problem with optimisation of operating conditions such as wall temperature and flow velocity in a comprehensive mitigation strategy while Ishiyama et al. (2010) considered the addition of the problem of controlling the desalter inlet temperature by using hot stream bypassing within a PHT fouling mitigation strategy based on heat exchanger cleaning.

Certain formulations include constraints set by pump-around operation (Smaïli et al., 2002a) and pressure drop (Smaïli et al., 2001), while both thermal and hydraulic impacts of fouling were considered by Ishiyama et al. (2009b) where variable throughput and control valve operation are implemented on the cleaning scheduling problem.

A cleaning operation will ideally remove all fouling deposits from a heat transfer surface. In practice the effectiveness of a cleaning operation depends on the nature of the deposit and the method of cleaning. Ishiyama et al. (2011) presented a framework for incorporating this complexity into the scheduling problem. The replacement of the single layer fouling model with a dual layer consisting of a soft exterior deposit (gel) and a harder interior layer (coke) was investigated by Pogiatzis et al. (2012). They considered the case where two cleaning methods were available: (a) cleaning-in-place methods and (b) off-line mechanical cleaning. An extra decision variable is added to the scheduling model, capturing the choice of cleaning method. The current paper addresses a single layer fouling model where the fouling kinetics exhibit linear and asymptotic behaviour.

Current solution methods for the cleaning scheduling problem still present limitations. Due to the complexity of networks and the nonlinearity in the models, MINLP approaches sometimes suffer from failure in convergence (Georgiadis and Papageorgiou, 2000; Smaïli et al., 2001) whereas MILP techniques may be computationally expensive (Lavaja and Bagajewicz, 2004) and involve the introduction of approximations to models. For example, Geor- 
giadis and Papageorgiou (2000) used the arithmetic temperature difference instead of the logarithmic mean temperature difference, which is not suitable for large networks that feature extensive feedback of hot (and/or cold) streams (Smaïli et al., 2001).

Stochastic optimisation methods may not be capable of handling problems involving many variables of similar effect (Fouskakis and Draper, 2002). Furthermore, these approaches can be very dependent on parameter tuning (Gonçalves et al., 2014). Solutions found by heuristic schemes such as greedy algorithms are not guaranteed to be optimal. For the scheduling problem, such simple strategies consider cleaning actions only in the current period and may be inefficient (Smaïli et al., 2001). Therefore, there is a need to develop robust, reliable and inexpensive methods to solve the scheduling cleaning problem.

In this paper we show for the first time that the heat exchanger network (HEN) cleaning scheduling problems are in actuality mixed-integer optimal control problems (MIOCPs) which exhibit a nearly bang-bang solution. This paper is arranged as follows: section 2 describes the formulation as a multi-period optimal control problem (OCP), including the proof of linearity of the control resulting in this bang-bang optimal solution behaviour. The formulation considered for the general HEN cleaning scheduling problem is presented in section 3. Implementation and solutions to a number of case studies for crude oil PHT obtained using a commercial optimisation software are presented in sections 4 and 5, including comparison of solutions to those produced through MP techniques.

\section{HEN Scheduling Optimisation as Multi-period Optimal Control}

This section will demonstrate that the HEN cleaning scheduling problem is in actuality a MIOCP. In this problem the controls, i.e. cleaning decisions occur linearly in the system, thus resulting in a bang-bang solution. Hence, integrality of the solution can be obtained by solving only the relaxed MIOCP as a standard nonlinear programming (NLP). Furthermore, proof of linearity in the control is shown in this section.

The basic formulation for an OCP is expressed in equations (1a) to (1d) where the per- 
112

113

114

115 116

117

subject to

$$
\min _{u(\cdot)} O=\phi\left[x\left(t_{\mathrm{F}}\right)\right]+\int_{0}^{t_{\mathrm{F}}} L[x(t), y(t), u(t), t] d t
$$

$$
u(t) \in \mathcal{U}, \quad \mathcal{U} \in\{0,1\} \quad \forall t \in\left[0, t_{\mathrm{F}}\right]
$$

$J_{p}\left(\dot{x}_{p+1}\left(t_{p}^{+}\right), x_{p+1}\left(t_{p}^{+}\right), y_{p+1}\left(t_{p}^{+}\right), u_{p+1}\left(t_{p}^{+}\right), \dot{x}_{p}\left(t_{p}^{-}\right), x_{p}\left(t_{p}^{-}\right), y_{p}\left(t_{p}^{-}\right), u_{p}\left(t_{p}^{-}\right), t_{p}\right)=0 \quad \forall p=1,2, \ldots, N P-1$

${ }_{123}$ The basic formulation of a multi-period OCP over time periods, $p=1, \ldots, N P, t \in$ ${ }_{124}\left[t_{p-1}, t_{p}\right]$ with $t_{N P}=t_{\mathrm{F}}$ is shown in equations $(3 \mathrm{a})$ to $(3 \mathrm{~g})$. 


$$
\min _{u(\cdot)} O=\sum_{p=1}^{N P}\left[\phi^{(p)} x\left(t_{p}\right), y^{(p)}\left(t_{p}\right), u^{(p)}, t^{(p)}\right]+\int_{t_{p-1}}^{t_{p}} L^{(p)}\left[x^{(p)}(t), y^{(p)}(t), u^{(p)}, t\right] d t
$$

125

subject to

$$
\begin{gathered}
\dot{x}^{(p)}(t)=f^{(p)}\left(x^{(p)}(t), y^{(p)}(t), u^{(p)}, t\right) \\
0=g^{(p)}\left(x^{(p)}(t), y^{(p)}(t), u^{(p)}, t\right) \\
t_{p-1} \leq t \leq t_{p}, \quad p=1,2, \ldots, N P \\
x^{(1)}\left(t_{0}\right)=I^{(1)}\left(u^{(1)}\right) \\
x^{(p)}\left(t_{p-1}\right)=I^{(p)}\left(x^{(p-1)}\left(t_{p-1}\right), y^{(p-1)}\left(t_{p-1}\right), u^{(p)}\right) \quad \forall p=2,3, \ldots, N P \\
u(t) \in \mathcal{U}, \quad \mathcal{U} \in\{0,1\}
\end{gathered}
$$

126 127 128

$$
\boldsymbol{u}=\left(\left(u^{(1)}\right)^{T},\left(u^{(2)}\right)^{T}, \ldots,\left(u^{(N P)}\right)^{T}\right)^{T}
$$

129 At the junctions, conditions are set where differential state variables are allowed to be reinitialised based on the control variable value: 


$$
x^{p}\left(t_{p-1}\right)=u^{p}(t) \cdot x^{p-1}\left(t_{p-1}\right) \quad \forall p=2, \ldots N P
$$

131 Proof that the control in the relaxed multistage MIOCP for cleaning scheduling is linearly

$$
\begin{aligned}
\bar{O}= & \sum_{p=2}^{N P}\{ \\
& \phi^{(p)}\left(x^{(p)}\left(t_{p}\right), y^{(p)}\left(t_{p}\right), u^{(p)}, t^{(p)}\right) \\
& +\left(\lambda^{(p)}\left(t_{p-1}\right)\right)^{T} \cdot\left(I^{(p)}\left(x^{(p-1)}\left(t_{p-1}\right), y^{(p-1)}\left(t_{p-1}\right), u^{(p)}\right)-x^{(p)}\left(t_{p-1}\right)\right) \\
& +\int_{t_{p-1}}^{t_{p}} L^{(p)}\left(x^{(p)}(t), y^{(p)}\left(t_{p}\right), u^{(p)}, t\right) d t \\
& +\int_{t_{p-1}}^{t_{p}}\left(\lambda^{(p)}(t)\right)^{T} \cdot\left(f^{(p)}\left(x^{(p)}(t), y^{(p)}\left(t_{p}\right), u^{(p)}, t\right)-\dot{x}^{(p)}(t)\right) d t \\
& +\int_{t_{p-1}}^{t_{p}}\left(\mu^{(p)}(t)\right)^{T} \cdot\left(g^{(p)}\left(x^{(p)}(t), y^{(p)}(t), u^{(p)}, t\right)\right) d t \\
& +{ }_{t_{0}}\left(\mu^{(1)}(t)\right)^{T} \cdot\left(g^{(1)}\left(x^{(1)}(t), y^{(1)}(t), u^{(1)}, t\right)\right) d t \\
& +\phi^{(1)}\left(x^{(1)}\left(t_{1}\right), y^{(1)}\left(t_{1}\right), u^{(1)}, t^{(1)}\right) \\
& +\left(\lambda^{(1)}\left(t_{0}\right)\right)^{T} \cdot\left(I^{(1)}\left(u^{(1)}\right)-x^{(1)}\left(t_{0}\right)\right) \\
& +\int_{t_{0}}^{t_{1}} L^{(1)}\left(x^{(1)}(t), y^{(1)}(t), u^{(1)}, t\right) d t \\
& +\int_{t_{0}}^{t_{1}}\left(\lambda^{(1)}(t)\right)^{T} \cdot\left(f^{(1)}\left(x^{(1)}(t), y^{(1)}(t), u^{(1)}, t\right)-\dot{x}^{(1)}(t)\right) d t \\
& +\int_{t_{1}}(t)
\end{aligned}
$$

Variations on the parameter set of stage $p^{\prime}$, of the form $\delta u^{\left(p^{\prime}\right)}$ are considered, which result in variations in the state values at all times as shown in equation (7). Clearly, the state vector 
138

$$
\begin{aligned}
& \delta \bar{O}=\sum_{p=2}^{N P}\{ \\
& {\left[\frac{\partial \phi^{(p)}}{\partial x^{(p)}\left(t_{p}\right)} \delta x^{(p)}\left(t_{p}\right)+\frac{\partial \phi^{(p)}}{\partial y^{(p)}\left(t_{p}\right)} \delta y^{(p)}\left(t_{p}\right)+\frac{\partial \phi^{(p)}}{\partial u^{(k)}} \delta u^{(p)}\right]} \\
& +\left(\lambda^{(p)}\left(t_{p-1}\right)\right)^{T} \text {. } \\
& \left(\frac{\partial I^{(p)}}{\partial x^{(p-1)}\left(t_{p-1}\right)} \delta x^{(p-1)}\left(t_{p-1}\right)+\frac{\partial I^{(p)}}{\partial y^{(p-1)}\left(t_{p-1}\right)} \delta y^{(p-1)}\left(t_{p-1}\right)+\frac{\partial I^{(p)}}{\partial u^{(p)}} \delta u^{(p)}-\delta x^{(p)}\left(t_{p-1}\right)\right) \\
& +\int_{t_{p-1}}^{t_{p}} \frac{\partial L^{(p)}}{\partial x^{(p)}(t)} \delta x^{(p)}(t)+\frac{\partial L^{(p)}}{\partial y^{(p)}(t)} \delta y^{(p)}(t)+\frac{\partial L^{(p)}}{\partial u^{(p)}} \delta u^{(p)} d t \\
& +\int_{t_{p-1}}^{t_{p}}\left(\lambda^{(p)}(t)\right)^{T} \cdot\left(\frac{\partial f^{(p)}}{\partial x^{(p)}(t)} \delta x^{(p)}(t)+\frac{\partial f^{(p)}}{\partial y^{(p)}(t)} \delta y^{(p)}(t)+\frac{\partial f^{(p)}}{\partial u^{(p)}} \delta u^{(p)}-\delta \dot{x}^{(p)}(t)\right) d t \\
& +\int_{t_{p-1}}^{t_{p}}\left(\mu^{(p)}(t)\right)^{T} \cdot\left(\frac{\partial g^{(p)}}{\partial x^{(p)}(t)} \delta x^{(p)}(t)+\frac{\partial g^{(p)}}{\partial y^{(p)}(t)} \delta y^{(p)}(t)+\frac{\partial g^{(p)}}{\partial u^{(p)}} \delta u^{(p)}\right) d t \\
& \} \\
& +\left[\frac{\partial \phi^{(1)}}{\partial x^{(1)}\left(t_{1}\right)} \delta x^{(1)}\left(t_{1}\right)+\frac{\partial \phi^{(1)}}{\partial y^{(1)}\left(t_{1}\right)} \delta y^{(1)}\left(t_{1}\right)+\frac{\partial \phi^{(1)}}{\partial u^{(1)}} \delta u^{(1)}\right] \\
& +\left(\lambda^{(1)}\left(t_{0}\right)\right)^{T} \cdot\left(\frac{\partial I^{(1)}}{\partial u^{(1)}} \delta u^{(1)}-\delta x^{(1)}\left(t_{0}\right)\right) \\
& +\int_{t_{0}}^{t_{1}} \frac{\partial L^{(1)}}{\partial x^{(1)}(t)} \delta x^{(1)}(t)+\frac{\partial L^{(1)}}{\partial y^{(1)}(t)} \delta y^{(1)}(t)+\frac{\partial L^{(1)}}{\partial u^{(1)}} \delta u^{(1)} d t \\
& +\int_{t_{0}}^{t_{1}}\left(\lambda^{(1)}(t)\right)^{T} \cdot\left(\frac{\partial f^{(1)}}{\partial x^{(1)}(t)} \delta x^{(1)}(t)+\frac{\partial f^{(1)}}{\partial y^{(1)}(t)} \delta y^{(1)}(t)+\frac{\partial f^{(1)}}{\partial u^{(1)}} \delta u^{(1)}-\delta \dot{x}^{(1)}(t)\right) d t \\
& +\int_{t_{0}}^{t_{1}}\left(\mu^{(1)}(t)\right)^{T} \cdot\left(\frac{\partial g^{(1)}}{\partial x^{(1)}(t)} \delta x^{(1)}(t)+\frac{\partial g^{(1)}}{\partial y^{(1)}(t)} \delta y^{(1)}(t)+\frac{\partial g^{(1)}}{\partial u^{(1)}} \delta u^{(1)}\right) d t
\end{aligned}
$$

139

Integration by parts for the last term in the integrals involving $\delta \dot{x}^{(p)}$ is used to obtain 140 equation (8): 


$$
\begin{aligned}
& \delta \bar{O}=\sum_{p=2}^{N P}\{ \\
& {\left[\frac{\partial \phi^{(p)}}{\partial x^{(p)}\left(t_{p}\right)} \delta x^{(p)}\left(t_{p}\right)+\frac{\partial \phi^{(p)}}{\partial y^{(p)}\left(t_{p}\right)} \delta y^{(p)}\left(t_{k}\right)+\frac{\partial \phi^{(p)}}{\partial u^{(p)}} \delta u^{(p)}\right]} \\
& +\left(\lambda^{(p)}\left(t_{p-1}\right)\right)^{T} \text {. } \\
& \left(\frac{\partial I^{(p)}}{\partial x^{(p-1)}\left(t_{p-1}\right)} \delta x^{(p-1)}\left(t_{p-1}\right)+\frac{\partial I^{(p)}}{\partial y^{(p-1)}\left(t_{p-1}\right)} \delta y^{(p-1)}\left(t_{p-1}\right)+\frac{\partial I^{(p)}}{\partial u^{(p)}} \delta u^{(p)}-\delta x^{(p)}\left(t_{p-1}\right)\right) \\
& +\int_{t_{p-1}}^{t_{p}} \frac{\partial L^{(p)}}{\partial x^{(p)}(t)} \delta x^{(p)}(t)+\frac{\partial L^{(p)}}{\partial y^{(p)}(t)} \delta y^{(p)}(t)+\frac{\partial L^{(p)}}{\partial u^{(p)}} \delta u^{(p)} d t \\
& +\int_{t_{p-1}}^{t_{p}}\left(\lambda^{(p)}(t)\right)^{T} \cdot\left(\frac{\partial f^{(p)}}{\partial x^{(p)}(t)} \delta x^{(p)}(t)+\frac{\partial f^{(p)}}{\partial y^{(p)}(t)} \delta y^{(p)}(t)+\frac{\partial f^{(p)}}{\partial u^{(p)}} \delta u^{(p)}\right) d t \\
& +\int_{t_{p-1}}^{t_{p}}\left(\dot{\lambda}^{(p)}(t)\right)^{T} \delta x^{(p)}(t) d t \\
& +\left(\lambda^{(p)}\left(t_{p-1}\right)\right)^{T} \cdot \delta x^{(p)}\left(t_{p-1}\right)-\left(\lambda^{(p)}\left(t_{p}\right)\right)^{T} \cdot \delta x^{(p)}\left(t_{p}\right) \\
& +\int_{t_{p-1}}^{t_{p}}\left(\mu^{(p)}(t)\right)^{T} \cdot\left(\frac{\partial g^{(p)}}{\partial x^{(p)}(t)} \delta x^{(p)}(t)+\frac{\partial g^{(p)}}{\partial y^{(p)}(t)} \delta y^{(p)}(t)+\frac{\partial g^{(p)}}{\partial u^{(p)}} \delta u^{(p)}\right) d t \\
& \} \\
& +\left[\frac{\partial \phi^{(1)}}{\partial x^{(1)}\left(t_{1}\right)} \delta x^{(1)}\left(t_{1}\right)+\frac{\partial \phi^{(1)}}{\partial y^{(1)}\left(t_{1}\right)} \delta y^{(1)}\left(t_{1}\right)+\frac{\partial \phi^{(1)}}{\partial u^{(1)}} \delta u^{(1)}\right] \\
& +\left(\lambda^{(1)}\left(t_{0}\right)\right)^{T} \cdot\left(\frac{\partial I^{(1)}}{\partial u^{(1)}} \delta u^{(1)}-\delta x^{(1)}\left(t_{0}\right)\right) \\
& +\int_{t_{0}}^{t_{1}} \frac{\partial L^{(1)}}{\partial x^{(1)}(t)} \delta x^{(1)}(t)+\frac{\partial L^{(1)}}{\partial y^{(1)}(t)} \delta y^{(1)}(t)+\frac{\partial L^{(1)}}{\partial u^{(1)}} \delta u^{(1)} d t \\
& +\int_{t_{0}}^{t_{1}}\left(\lambda^{(1)}(t)\right)^{T} \cdot\left(\frac{\partial f^{(1)}}{\partial x^{(1)}(t)} \delta x^{(1)}(t)+\frac{\partial f^{(1)}}{\partial y^{(1)}(t)} \delta y^{(1)}(t)+\frac{\partial f^{(1)}}{\partial u^{(1)}} \delta u^{(1)}\right) d t \\
& +\int_{t_{0}}^{t_{1}}\left(\dot{\lambda}^{(1)}(t)\right)^{T} \delta x^{(1)}(t) d t \\
& +\left(\lambda^{(1)}\left(t_{0}\right)\right)^{T} \cdot \delta x^{(1)}\left(t_{0}\right)-\left(\lambda^{(1)}\left(t_{1}\right)\right)^{T} \cdot \delta x^{(1)}\left(t_{1}\right) \\
& +\int_{t_{0}}^{t_{1}}\left(\mu^{(1)}(t)\right)^{T} \cdot\left(\frac{\partial g^{(1)}}{\partial x^{(1)}(t)} \delta x^{(1)}(t)+\frac{\partial g^{(1)}}{\partial y^{(1)}(t)} \delta y^{(1)}(t)+\frac{\partial g^{(1)}}{\partial u^{(1)}} \delta u^{(1)}\right) d t
\end{aligned}
$$

141 For a stationary point, infinitesimal variations in the right hand side should yield no 142 change to the performance index, i.e. $\delta \bar{O}=0$, and hence related terms must be chosen so 
143

144 145 146 conditions as shown in equations (9a) to (10) must hold, respectively:

$$
\begin{gathered}
\dot{\lambda}^{(1)}(t)=-\left[\frac{\partial f^{(1)}}{\partial x^{(1)}(t)}\right]^{T} \lambda^{(1)}(t)-\left[\frac{\partial g^{(1)}}{\partial x^{(1)}(t)}\right]^{T} \mu^{(1)}(t)-\left[\frac{\partial L^{(1)}}{\partial x^{(1)}(t)}\right]^{T} \\
t_{0} \leq t \leq t_{1} \\
\lambda^{(1)}\left(t_{1}\right)=\left[\frac{\partial \phi^{(1)}}{\partial x^{(1)}\left(t_{1}\right)}\right]^{T}
\end{gathered}
$$

147 Algebraic equations and final stage conditions (11a) to (11b) must hold in order to cancel 148 the $\delta y^{(1)}(t)$ and $\delta y^{(1)}\left(t_{1}\right)$ terms;

$$
\begin{gathered}
{\left[\frac{\partial f^{(1)}}{\partial y^{(1)}(t)}\right]^{T} \lambda^{(1)}(t)+\left[\frac{\partial g^{(1)}}{\partial y^{(1)}(t)}\right]^{T} \mu^{(1)}(t)+\left[\frac{\partial L^{(1)}}{\partial y^{(1)}(t)}\right]^{T}=0} \\
t_{0} \leq t \leq t_{1} \\
{\left[\frac{\partial \phi^{(1)}}{\partial y^{(1)}\left(t_{1}\right)}\right]^{T}+\left[\frac{\partial I^{(2)}}{\partial y^{(1)}\left(t_{1}\right)}\right]^{T} \cdot \lambda^{(2)}\left(t_{1}\right)=0}
\end{gathered}
$$

149 The $\delta x^{(p)}(t)$ and $\delta x^{(p)}\left(t_{p}\right)$ terms are cancelled through the condition that the following dif150 ferential equations and final time stage conditions are held;

$$
\dot{\lambda}^{(p)}(t)=-\left[\frac{\partial f^{(p)}}{\partial x^{(p)}(t)}\right]^{T} \lambda^{(p)}(t)-\left[\frac{\partial L^{(p)}}{\partial x^{(p)}(t)}\right]^{T}
$$

151

$$
t_{p-1} \leq t \leq t_{p} \quad \forall p=2,3, \ldots N P
$$




$$
\lambda^{(p)}\left(t_{p}\right)=\left[\frac{\partial \phi^{(p)}}{\partial x^{(p)}\left(t_{p}\right)}\right]^{T}+\left[\frac{\partial I^{(p+1)}}{\partial x^{(p)}\left(t_{p}\right)}\right]^{T} \cdot \lambda^{(p+1)}\left(t_{p}\right) \quad \forall p=2,3, \ldots, N P-1
$$

152 To cancel $\delta y^{(p)}(t)$ and $\delta y^{(p)}\left(t_{p}\right)$ terms, the following algebraic equations must hold:

$$
\begin{gathered}
{\left[\frac{\partial f^{(p)}}{\partial y^{(p)}(t)}\right]^{T} \lambda^{(p)}(t)+\left[\frac{\partial g^{(p)}}{\partial y^{(p)}(t)}\right]^{T} \mu^{(p)}(t)+\left[\frac{\partial L^{(p)}}{\partial y^{(p)}(t)}\right]^{T}=0} \\
t_{p-1} \leq t \leq t_{p} \quad \forall p=2,3, \ldots N P \\
{\left[\frac{\partial \phi^{(p)}}{\partial y^{(p)}\left(t_{p}\right)}\right]^{T}+\left[\frac{\partial I^{(p+1)}}{\partial y^{(p)}\left(t_{p}\right)}\right]^{T} \cdot \lambda^{(p+1)}\left(t_{p}\right)=0 \quad \forall p=2,3, \ldots, N P-1}
\end{gathered}
$$

153 The terms $\delta u^{(1)}$ and $\delta u^{(p)}$ are cancelled on the condition that equations (15a) to (16b) hold.

154 These are equivalent to the Hamiltonian gradient condition:

$$
\begin{aligned}
& {\left[\frac{\partial \phi^{(1)}}{\partial u(1)}\left(t_{1}\right)\right]^{T}+\left[\frac{\partial I^{(1)}}{\partial u^{(1)}}\right]^{T} \cdot \lambda^{(1)}\left(t_{0}\right)} \\
& +\int_{t_{0}}^{t_{1}}\left\{\left[\frac{\partial L^{(1)}}{\partial u^{(1)}}(t)\right]^{T}+\left[\frac{\partial f^{(1)}}{\partial u^{(1)}}(t)\right]^{T} \cdot \lambda^{(1)}(t)+\left[\frac{\partial g^{(1)}}{\partial u^{(1)}}(t)\right]^{T} \cdot \mu^{(1)}(t)\right\} d t=0
\end{aligned}
$$$$
t_{0} \leq t \leq t_{1}
$$

$$
\begin{aligned}
& {\left[\frac{\partial \phi^{(p)}}{\partial u^{(p)}}\left(t_{p}\right)\right]^{T}+\left[\frac{\partial I^{(p)}}{\partial u^{(p)}}\right]^{T} \cdot \lambda^{(p)}\left(t_{p-1}\right)} \\
& +\int_{t_{p-1}}^{t_{p}}\left\{\left[\frac{\partial L^{(p)}}{\partial u^{(p)}}(t)\right]^{T}+\left[\frac{\partial f^{(p)}}{\partial u^{(p)}}(t)\right]^{T} \cdot \lambda^{(p)}(t)+\left[\frac{\partial g^{(p)}}{\partial u^{(p)}}(t)\right]^{T} \cdot \mu^{(p)}(t)\right\} d t=0
\end{aligned}
$$




$$
t_{p-1} \leq t \leq t_{p} \quad \forall p=2,3, \ldots N P
$$

When the functions appearing in equations (15a) and (16a) are linearly related to the control, the optimal control for the relaxed MIOCP will exhibit bang-bang behaviour (with potential singular arcs). Bang-bang solutions occur when the optimal control action is at either bound of the feasible region (Bryson and Ho, 1975). Controls that are not bangbang, where the control lies between the bounds, are called singular. In this case, singular arcs exist. Pure bang-bang controls are demonstrated in minimum-time problems for linear systems (Bellman et al., 1956) and bilinear systems (Mohler, 1973), optimal control of batch reactors (Blakemore and Aris, 1962), optimal thermal control (Belghith et al., 1986), etc.

For nonlinear optimisation systems, this bang-bang principle does not always hold. Zandvliet et al. (2007) investigated reservoir flooding problems, where the control is linear in relation to the continuous variables, and showed that if the only constraints are upper and lower bounds on the control, then due to their particular structure, these problems will sometimes have bang-bang optimal solutions. This is advantageous since bang-bang solutions can be implemented with simple on-off control valves.

Approaches for optimal control of nonlinear dynamical systems with binary controls (on/off) were reviewed by Sager (2009). To satisfy requirements for bang-bang behaviour, the general OCP is reformulated such that the binary controls are presented linearly in the system dynamics. Solutions in this case may require use of heuristics e.g. rounding up or a sum up rounding strategy, or algorithms such as Branch and Bound when singular arcs appear (Sager, 2009).

For the scheduling cleaning problem, reformulation is not necessary as the controls involved already have linear presentation in the system. More importantly, the formulation of this problem as an OCP facilitates the solution of the relaxed nonlinear programming (NLP) problem through the feasible path approach, obviating the need to discretise the system equations. This otherwise leads to a very large scale optimisation problem with a strongly 
nonlinear system of equality constraints. This approach avoids failures of convergence produced by direct solutions of MINLPs resulting from discretisation, such as in previous work of Georgiadis and Papageorgiou (2000) and of Smaïli et al. (2001).

\section{HEN Scheduling Optimisation Formulation}

The effect of fouling on heat transfer performance is often quantified in lumped parameter models of process heat transfer via the fouling resistance, $R_{\mathrm{f}}$.

$$
\frac{1}{U}=\frac{1}{U_{\mathrm{c}}}+R_{\mathrm{f}}
$$

Equation (17) expresses the overall heat transfer coefficient $U$ in relation to the fouling resistance and $U_{\mathrm{c}}$, its value when clean.

The impact of fouling resistance is more severe for heat exchangers with a high overall heat transfer coefficient. Both linear (equation (18)) and exponentially asymptotic fouling behaviour (equation (19)) are considered in this paper, which are quantified via

$$
\begin{gathered}
\dot{R}_{\mathrm{f}}=a \\
R_{\mathrm{f}}=R_{\mathrm{f}}^{\infty}\left(1-\exp \left(-t^{\prime} / \tau\right)\right)
\end{gathered}
$$

where $a$ is the linear fouling constant for a particular heat exchanger, $R_{f}^{\infty}$ is the asymptotic fouling resistance, $\tau$ is the decay constant, and $t^{\prime}$ is the operating time elapsed since the last cleaning action.

The heat duty of a single-pass shell and tube heat exchanger operating in counter-current mode is given by equation (20), which is based on the log-mean temperature difference method.

$$
Q=U A \triangle T_{\mathrm{lm}}
$$

Here $A$ is the area and $\triangle T_{l m}$ is the logarithmic mean temperature difference. 
203

The heat duty, $Q$, is also linearly related to the stream inlet and outlet temperatures through the energy balances outlined in equations (21) and (22):

$$
Q=F_{\mathrm{c}} C_{\mathrm{c}}\left(T_{\mathrm{c}}^{\mathrm{out}}-T_{\mathrm{c}}^{\mathrm{in}}\right)
$$

$$
Q=F_{\mathrm{h}} C_{\mathrm{h}}\left(T_{\mathrm{h}}^{\mathrm{in}}-T_{\mathrm{h}}^{\mathrm{out}}\right)
$$

where $F_{\mathrm{h}}$ and $F_{\mathrm{c}}$ are the mass flow-rates of the hot and cold streams respectively, and $C_{\mathrm{h}}$ and $C_{\mathrm{c}}$ are their specific heat capacities.

The cleaning scheduling problem is a multi-period OCP where a decision must be made regarding when, i.e. in which period(s), cleaning should occur, and which unit is to be cleaned. The control action is discretised into time periods of equal length, where each period is discretised further into a cleaning and operating sub-period. This is represented by binary variable $y_{n p}$ which is used to describe the cleaning status of each exchanger in each cleaning sub-period, where

$$
y_{n p}=\left\{\begin{array}{cc}
0 & \text { if the } n \text {th heat exchanger is cleaned in period } p \\
1 & \text { otherwise }
\end{array}\right\} \forall n, p
$$

Within an operating sub-period, this binary variable is fixed to 1 for all $n$ i.e. all units are online. The objective is to minimise the operating and cleaning costs due to fouling over 4 a specified horizon of time $t_{\mathrm{F}}$. The objective function is given by equation (24). The form of this objective function is generally common to all approaches. Local considerations may give slightly different mathematical expressions. However, the differences lie in the solution approach.

$$
O b j=\int_{0}^{t_{\mathrm{F}}} \frac{C_{\mathrm{E}} Q_{\mathrm{F}}(t)}{\eta_{f}} d t+\sum_{p=1}^{N P} \sum_{n=1}^{N E} C_{\mathrm{c}}\left(1-y_{n p}\right)
$$

219 The extra furnace energy consumption is described by the term $Q_{\mathrm{F}}(t)$ which is determined based on the temperature of the crude oil entering the furnace, i.e. the crude inlet temperature (CIT). $C_{\mathrm{E}}$ represents the cost of fuel, $\eta_{f}$ is the furnace efficiency, $N E$ is the number 
incorporation of binary variable $y_{n p}$, equations (18) and (19) can be rewritten as:

$$
\begin{gathered}
\dot{R}_{\mathrm{f}}=y_{n p} a \quad \forall n, p \\
R_{\mathrm{f}}=R_{\mathrm{f}}^{\infty}\left(1-\exp \left(-t^{\prime} / \tau\right)\right) \\
\dot{t}^{\prime}=y_{n p} \quad \forall n, p
\end{gathered}
$$

231

232

233

234

235

236

237

238

239

249 are reproduced from Smaïli et al. (2001) :

$$
\alpha=\frac{U A}{F_{\mathrm{h}} C_{\mathrm{h}}}
$$




$$
P=\frac{F_{\mathrm{h}} C_{\mathrm{h}}}{F_{\mathrm{c}} C_{\mathrm{c}}}
$$

242 Through combination and rearrangement of equations (20), (21) and (22) the temperature

$$
\begin{aligned}
T_{\mathrm{h}}^{\text {out }}= & y_{n p}\left[\frac{(1-P) T_{\mathrm{h}}^{\text {in }} \exp (-\alpha(1-P))+T_{\mathrm{c}}^{\text {in }}(1-\exp (-\alpha(1-P)))}{1-P \exp (-\alpha(1-P))}\right] \\
& +\left(1-y_{n p}\right) T_{\mathrm{h}}^{\text {in }} \quad \forall n, p
\end{aligned}
$$

\section{Implementation}

The implementation is performed in MATLAB ${ }^{\circledR}$ R2016b with its Optimisation Toolbox ${ }^{\mathrm{TM}}$ and Parallel Computing Toolbox ${ }^{\mathrm{TM}}$ (The MathWorks Inc., 2016). It is noteworthy that this methodology cannot be implemented in current commercial simulators directly. For example, gPROMS $^{\mathrm{TM}}$ (Process Systems Enterprise, 2017), which is one of the most advanced commercial simulators, does not facilitate multi-period optimal control problem solutions as it does not allow for junction conditions.

The MATLAB ${ }^{\circledR}$ code works as a standard multi-period optimal control problem solver using the feasible path approach (i.e. sequential approach) by linking together the Ordinary Differential Equation (ODE) solver ode15s with the optimiser fmincon. The default settings for ode15s are used, with absolute tolerance of $10^{-6}$ and relative tolerance of $10^{-3}$. The optimiser fmincon is used with the Sequential Quadratic Programming (SQP) algorithm option whilst keeping the remaining settings at their default values: constraint, optimality and step tolerances of $10^{-6}$ using a forward finite difference scheme for the estimation of gradi- 
ents. Gradient evaluations conducted via finite differences are costly and require repeated simulations of the dynamic process model.

Additionally, since this problem is non-convex, multiple runs with different starting points are performed and the best solution is reported. A test was run using the Parallel Computing Toolbox $^{\mathrm{TM}}$ to compare the computational time between parallelisation of the gradient evaluations versus parallelising a loop of multiple starting points. On a 4GHz Intel Core i7, 16 GB RAM iMac running on macOS Sierra the latter was faster than the former. Parallelisation of a loop of 50 runs is performed using a parfor loop. For cases where singularities appear in the control, a rounding up scheme is employed.

\section{Case Studies}

Computation experiments for the scheduling of cleaning actions for HENs located in crude oil distillation unit PHTs undergoing fouling are considered here. We present case studies appearing in the work of Lavaja and Bagajewicz (2004): a single heat exchanger unit; 4 units in series, a network of 10 units; and the more complex network of 25 units presented by Smaïli et al. (2002a). These are shown in figures 1 to 3. Stream data for each model are presented in tables 1 to 3 and 5 . For the 10 unit HEN case study presented in the Lavaja and Bagajewicz (2004) formulation and the 25 unit HEN case study presented in the Smaïli et al. (2002a) formulation, the selection and operational constraints imposed through consideration of performance targets or acceptable operating practice are shown in tables 4 and 6, respectively. These constraints are based only on exchanger cleaning actions. However, in practice temperature bounds on the performance of exchangers are required to be applied, for example in the case of desalter temperature control considered by Ishiyama et al. (2010). For the purpose of achieving results that can be compared to published ones from case studies in the open literature, only the constraints shown in tables 4 and 6 are imposed on the corresponding case studies.

The number of periods considered is $N P=24$ for the single unit and $N P=18$ for the 
10 unit HEN case studies while this is $N P=\{12,18\}$ for the 4 unit heat exchanger case study. A longer duration is considered for the 25 unit HEN, with $N P=36$. Both linear and asymptotic fouling models are considered in the single unit and 10 unit HEN cases whilst only linear fouling is modelled in the 4 units and 25 unit HEN case studies. This is done for comparison purposes.

The extra energy cost required due to fouling $C_{E}$ in the objective function displayed in equation (24) is $£ 0.34 / \mathrm{kW}$ day for the 25 unit HEN case. There is no mention of the furnace fuel cost in the work of Lavaja and Bagajewicz, so a cost of $22.93 / \mathrm{MM}$ Btu is used here based on the value reported by Smaïli et al. (2002b). The work of Smaïli et al. is the source of data for Lavaja and Bagajewicz's models where they compared the solutions from their MILP approach with those obtained by Smaili et al. using the OA/ER algorithm. Although Lavaja and Bagajewicz stated that they accounted for the decay in the heat transfer coefficient in each sub-period, expressed by $\eta_{c}$, there is no mention of the value of this parameter in their work. Hence, we considered the value of parameter $\eta_{c}$ to be 1 in our model. This decay parameter is also fixed at the value of 1 in the 25 unit HEN case study along with the furnace efficiency $\eta_{f}$. Smaïli et al. (2002b) did not consider these parameters in their model. The cleaning cost incurred for cleaning operations, $C_{c}$, is $£ 5000$ per cleaning action in the 25 unit HEN case and $£ 4000$ for all other cases. For the former case, the duration of the cleaning and operating sub-periods are equal with $\Delta t^{\mathrm{cl}}=\Delta t^{\mathrm{op}}=15$ days. If the cleaning time did depend on the size of the exchanger, these durations would have to be unit dependent.

The scheduling problem was reformulated into a MILP problem by Lavaja and Bagajewicz (2004) whereas Smailli et al. (2002a) solved the MINLP problem directly using two methods: a Backtracking Threshold Accepting (BTA) algorithm and the Outer Approximation (OA) method. 


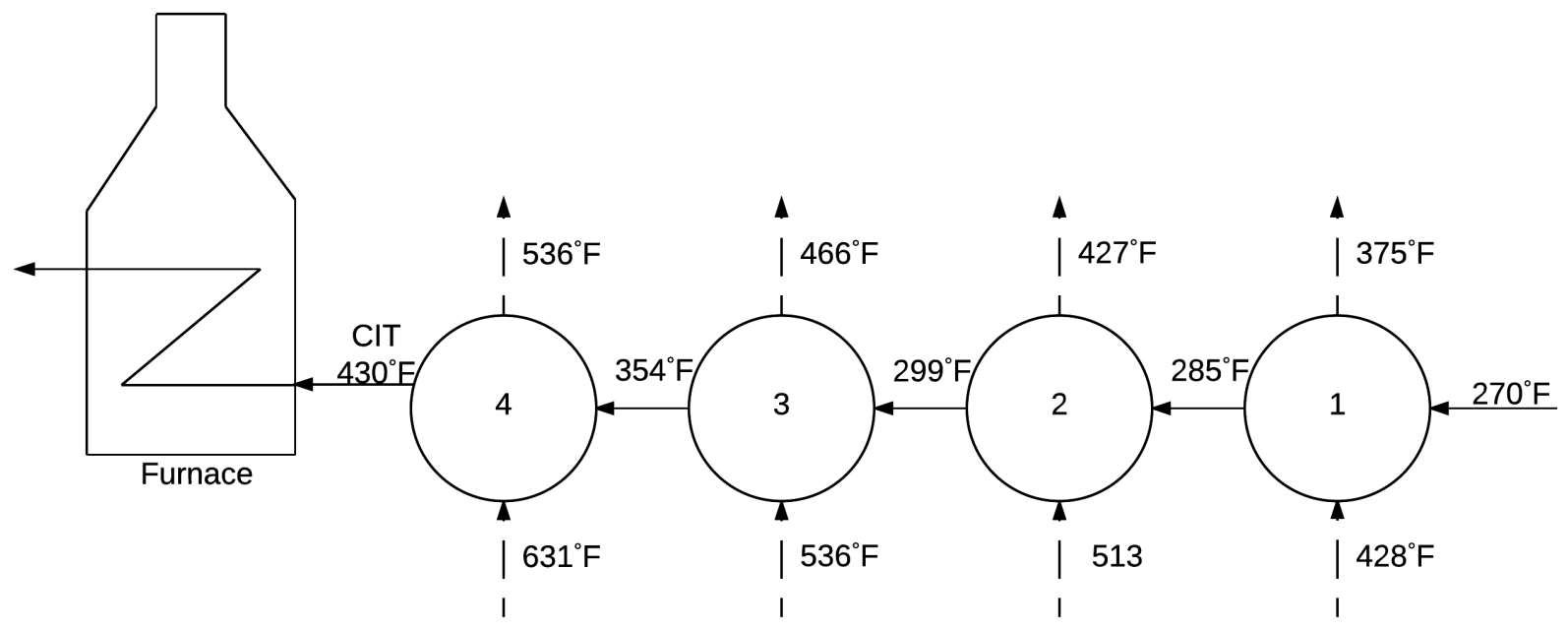

Figure 1: Four heat exchanger case. Temperature values are given for initial, clean condition. Adapted from Lavaja and Bagajewicz, 2004.

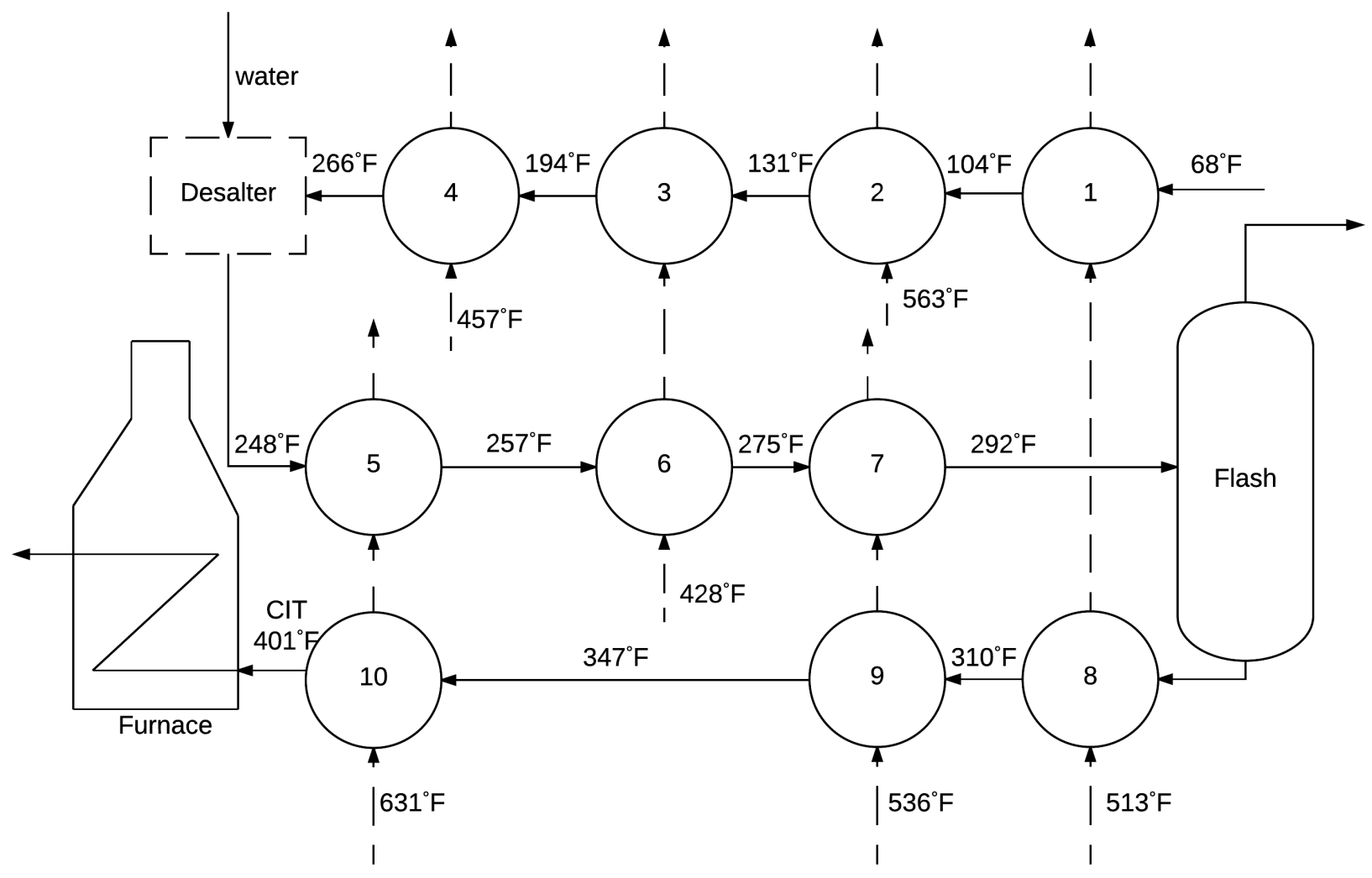

Figure 2: 10 unit HEN case. Temperature values are given for initial, clean condition. Adapted from Lavaja and Bagajewicz, 2004. 


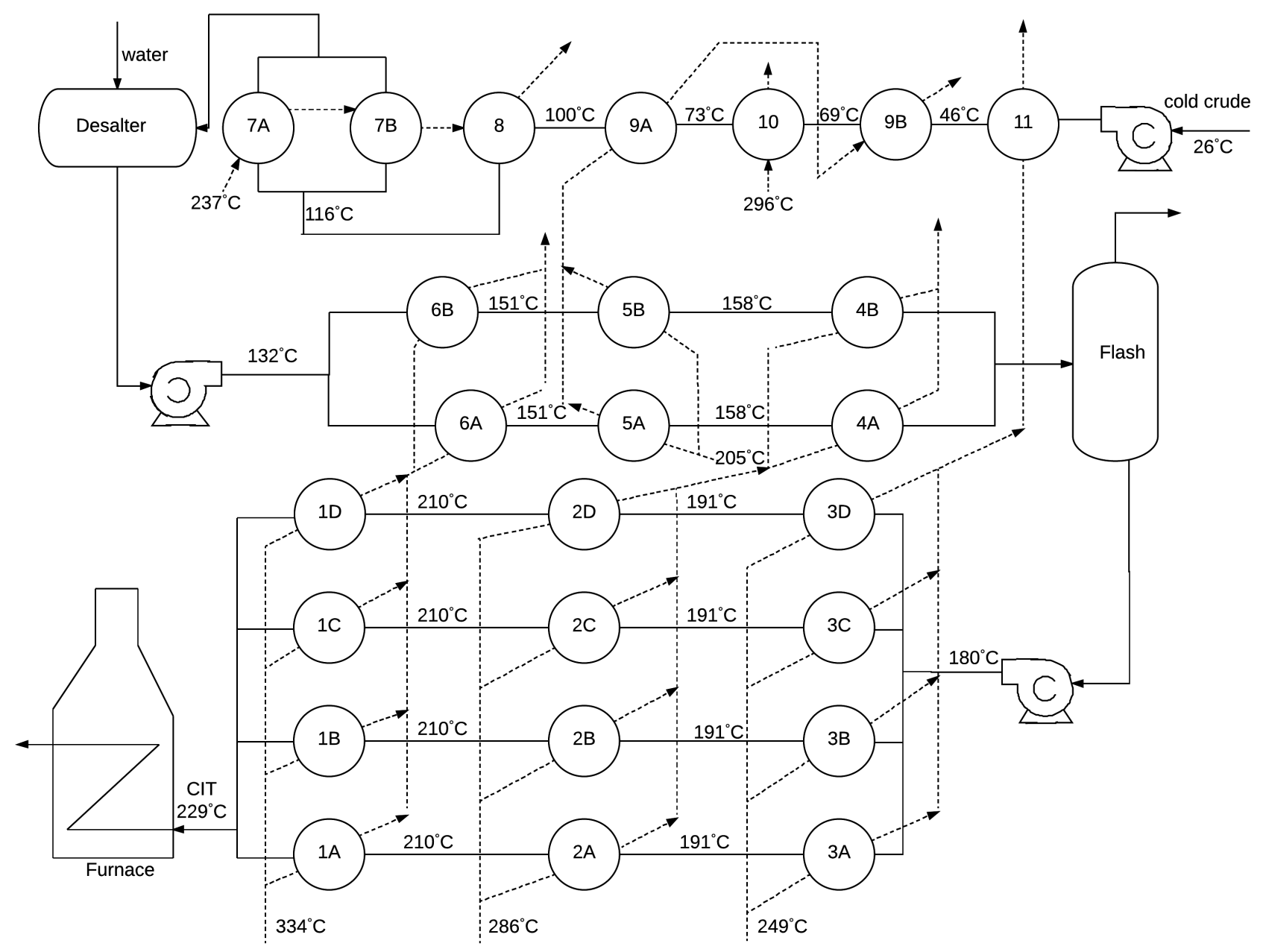

Figure 3: 25 unit HEN case. Solid lines, cold (crude) streams; dashed lines, hot streams; CIT, crude inlet temperature to furnace. Temperature values are given for initial, clean condition. Adapted from Smaïli et al., 2002a.

The cleaning schedules featuring the best objective, i.e. lowest overall cost, are reported for each case. The optimal cleaning schedules are presented in tables 11 to 16 alongside those obtained by Lavaja and Bagajewicz (2004) and Smaïli et al. (2002a). In the economic comparison, we placed the cleaning schedules obtained by Lavaja and Bagajewicz (2004) and Smaïli et al. (2002a) into our model to evaluate the cost. Tables 7 to 10 show the economic comparison.

Fouling rates directly impact the performance of heat exchangers. The asymptotic fouling cases have larger initial fouling rates, causing a rapid decay in the hot stream temperatures through the network, resulting in a much larger objective value for the uncleaned case (e.g. 
Table 1: Data for single heat exchanger case. Adapted from Lavaja and Bagajewicz, 2004.

\begin{tabular}{|ll|}
\hline Parameter & Value \\
$F_{\mathrm{h}}[\mathrm{lb} / \mathrm{h}]$ & 208000 \\
$F_{\mathrm{c}}[\mathrm{lb} / \mathrm{h}]$ & 649000 \\
$C_{\mathrm{h}}\left[\mathrm{Btu} / \mathrm{lb}{ }^{\circ} \mathrm{F}\right]$ & 0.67 \\
$C_{\mathrm{c}}\left[\mathrm{Btu} / \mathrm{lb}{ }^{\circ} \mathrm{F}\right]$ & 0.57 \\
$U_{\mathrm{c}}\left[\mathrm{Btu} / \mathrm{hft}^{2 \circ} \mathrm{F}\right]$ & 88.1 \\
$U_{0}\left[\mathrm{Btu} / \mathrm{hft}^{\circ} \mathrm{F}\right]$ & 88.1 \\
$A\left[\mathrm{ft}^{2}\right]$ & 1257 \\
$a(\mathrm{linear}$ fouling $)\left[\mathrm{ft}^{2 \circ} \mathrm{F} / \mathrm{Btu}\right]$ & $3.88 \times 10^{-7}$ \\
$R_{f}^{\infty}($ asymptotic fouling $)\left[\mathrm{hft}{ }^{\circ} \mathrm{F} / \mathrm{Btu}\right]$ & $6.73 \times 10^{-3}$ \\
$\tau(\mathrm{decay}$ constant $)[\mathrm{month}]$ & 4 \\
$\Delta t^{\mathrm{cl}}[$ month] & 0.20 \\
$\Delta t^{\mathrm{op}}[$ month $]$ & 0.80 \\
$\eta_{f}$ & 0.75 \\
\hline
\end{tabular}

$£ 317$ k vs. $2203 k$ for the single unit case shown in table 7). Consequently, one would expect more cleaning actions in all the asymptotic fouling model cases than the corresponding linear ones due to the early loss of exchanger efficiencies. This is evident in table 11 with the cleaning actions increasing from 3 to 5 in both this work's solution and the solution of Lavaja and Bagajewicz (2004). Similar observations to Lavaja and Bagajewicz (2004) are seen in the single unit case, where cleaning actions are cyclic (table 11). For linear fouling, the number of cleaning actions as well as the schedules are very similar: however the cleanings in our model are performed 1 month earlier than in Lavaja and Bagajewicz 's schedule.

For the four heat exchanger case, the number of cleaning actions are the same as Lavaja and Bagajewicz 's model and the schedule for the 12 month operating horizon is the same, meanwhile the schedule for the 18 month duration differs. No pattern is evident when the schedules are compared, with some cleaning actions occurring earlier in some cases and later in others.

In the majority of our cases our model produced similar overall costs to those reported by Lavaja and Bagajewicz (2004), the only differences being (i) the 4 heat exchanger case over 18 months, where the cost of our schedule is slightly smaller than that reported, with the difference in savings being only $<1.5 \%$; and (ii) the 10 unit HEN case with asymptotic 


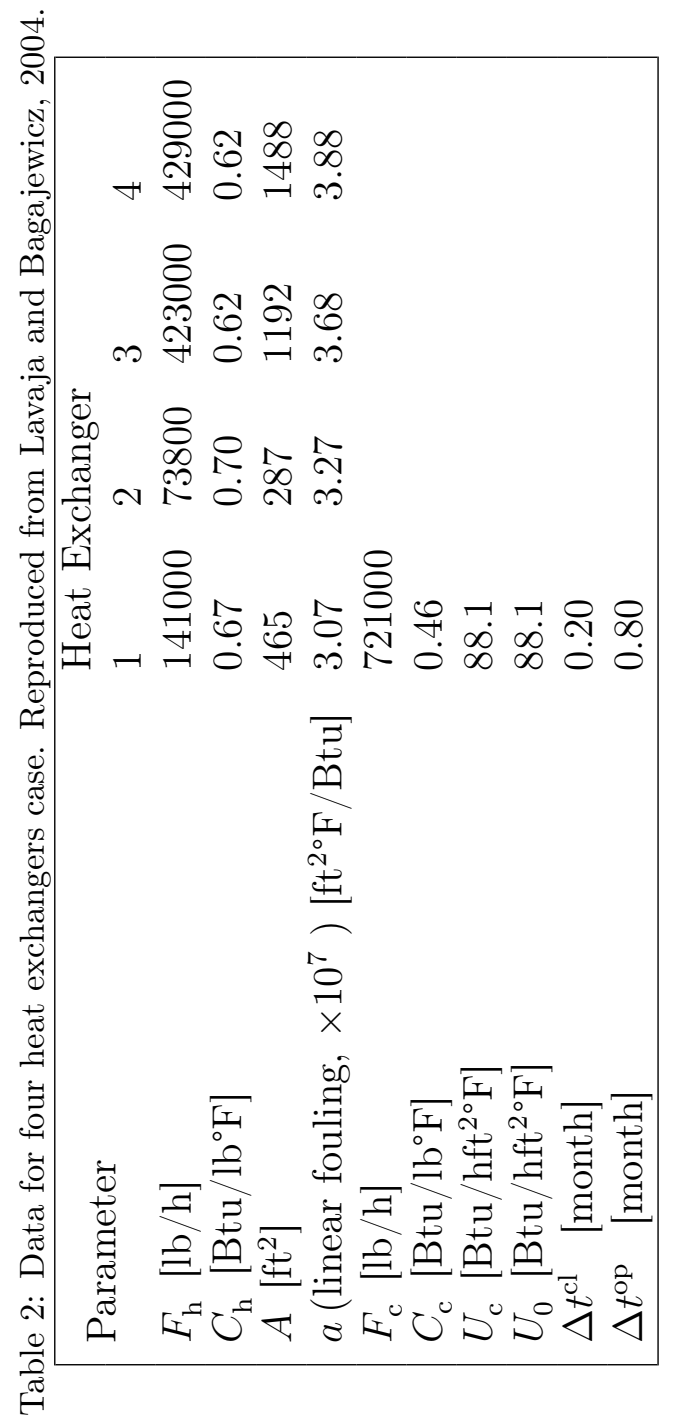




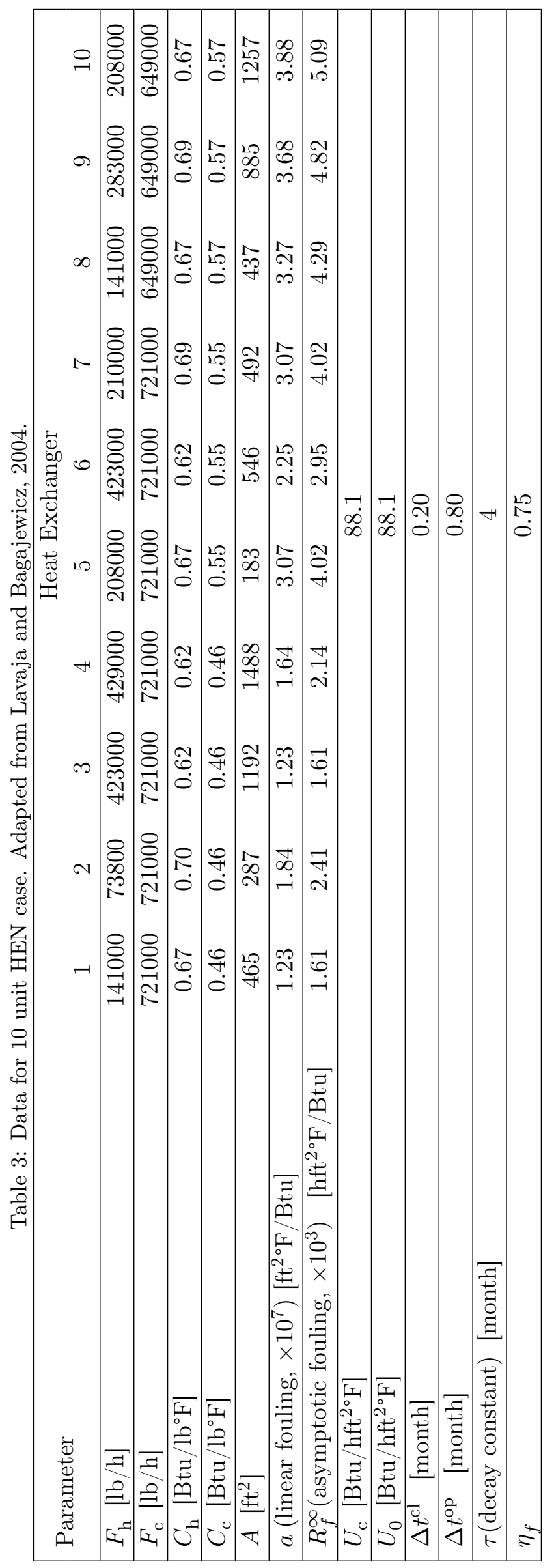


Table 4: Operational constraints for 10 unit HEN case.

$\begin{array}{lc}\text { only one unit of exchangers } 1-4 \text { can be cleaned in each period } & y_{1 p}+y_{2 p}+y_{3 p}+y_{4 p} \geq 3 \forall p \\ \text { only one unit of exchangers } 5-7 \text { can be cleaned in each period } & y_{5 p}+y_{6 p}+y_{7 p} \geq 2 \forall p \\ \text { temperature drop across desalter } & T_{c, 5 p}^{\text {in }}=T_{c, 4 p}^{\text {out }}-18 \forall p\end{array}$

Table 5: Data for 25 unit HEN case. Adapted from Smaïli et al., 2002a.

\begin{tabular}{|llllllll|}
\hline$H E X$ & $\begin{array}{l}F_{\mathrm{h}} \\
\left(\mathrm{kg} \mathrm{s}^{-1}\right)\end{array}$ & $\begin{array}{l}F_{\mathrm{c}} \\
\left(\mathrm{kg} \mathrm{s}^{-1}\right)\end{array}$ & $\begin{array}{l}C_{\mathrm{h}} \\
\left(\mathrm{kJ} \mathrm{kg}^{-1} \mathrm{~K}^{-1}\right)\end{array}$ & $\begin{array}{l}C_{\mathrm{c}} \\
\left(\mathrm{kJ} \mathrm{kg}^{-1} \mathrm{~K}^{-1}\right)\end{array}$ & $\begin{array}{l}U_{\mathrm{c}} \\
\left(\mathrm{kW} \mathrm{m}^{-2} \mathrm{~K}^{-1}\right)\end{array}$ & $\begin{array}{l}A \\
\left(\mathrm{~m}^{2}\right)\end{array}$ & $\begin{array}{l}a \times 10^{11} \\
\left(\mathrm{~m}^{2} \mathrm{KJ}^{-1}\right)\end{array}$ \\
\hline 1A & 8.7 & 23 & 2.8 & 2.4 & 0.5 & 21.3 & 1.9 \\
\hline 2A & 11.4 & 23 & 2.9 & 2.4 & 0.5 & 29.7 & 1.8 \\
\hline 3A & 4.8 & 23 & 2.8 & 2.4 & 0.5 & 31.4 & 1.6 \\
\hline 1B & 8.7 & 23 & 2.8 & 2.4 & 0.5 & 21.3 & 1.9 \\
\hline 2B & 11.4 & 23 & 2.9 & 2.4 & 0.5 & 29.7 & 1.8 \\
\hline 3B & 4.8 & 23 & 2.8 & 2.4 & 0.5 & 31.4 & 1.6 \\
\hline 1C & 8.7 & 23 & 2.8 & 2.4 & 0.5 & 21.3 & 1.9 \\
\hline 2C & 11.4 & 23 & 2.9 & 2.4 & 0.5 & 29.7 & 1.8 \\
\hline 3C & 4.8 & 23 & 2.8 & 2.4 & 0.5 & 31.4 & 1.6 \\
\hline 1D & 8.7 & 23 & 2.8 & 2.4 & 0.5 & 21.3 & 1.9 \\
\hline 2D & 11.4 & 23 & 2.9 & 2.4 & 0.5 & 29.7 & 1.8 \\
\hline 3D & 4.8 & 23 & 2.8 & 2.4 & 0.5 & 31.4 & 1.6 \\
\hline 4A & 23 & 47.4 & 2.8 & 2.3 & 0.5 & 26.7 & 1.5 \\
\hline 5A & 28 & 47.4 & 2.6 & 2.3 & 0.5 & 35.4 & 1.1 \\
\hline 6A & 17.4 & 47.4 & 2.9 & 2.3 & 0.5 & 79.1 & 1.5 \\
\hline 4B & 23 & 47.4 & 2.8 & 2.3 & 0.5 & 29.2 & 1.6 \\
\hline 5B & 28 & 47.4 & 2.6 & 2.3 & 0.5 & 35.4 & 1.1 \\
\hline 6B & 17.4 & 47.4 & 2.9 & 2.3 & 0.5 & 79.1 & 1.5 \\
\hline 7A & 25 & 47.4 & 2.6 & 1.92 & 0.5 & 60.8 & 0.8 \\
\hline 7B & 25 & 47.4 & 2.6 & 1.92 & 0.5 & 80.3 & 0.8 \\
\hline 8 & 49.6 & 95 & 2.6 & 1.92 & 0.5 & 129 & 0.8 \\
\hline 9A & 55.8 & 95 & 2.6 & 1.92 & 0.5 & 110 & 0.9 \\
\hline 9B & 55.8 & 95 & 2.6 & 1.92 & 0.5 & 96.6 & 0.9 \\
\hline 10 & 3.3 & 95 & 2.9 & 1.92 & 0.5 & 8.5 & 0.6 \\
\hline 11 & 19.1 & 95 & 2.8 & 1.92 & 0.5 & 56.6 & 0.6 \\
\hline
\end{tabular}




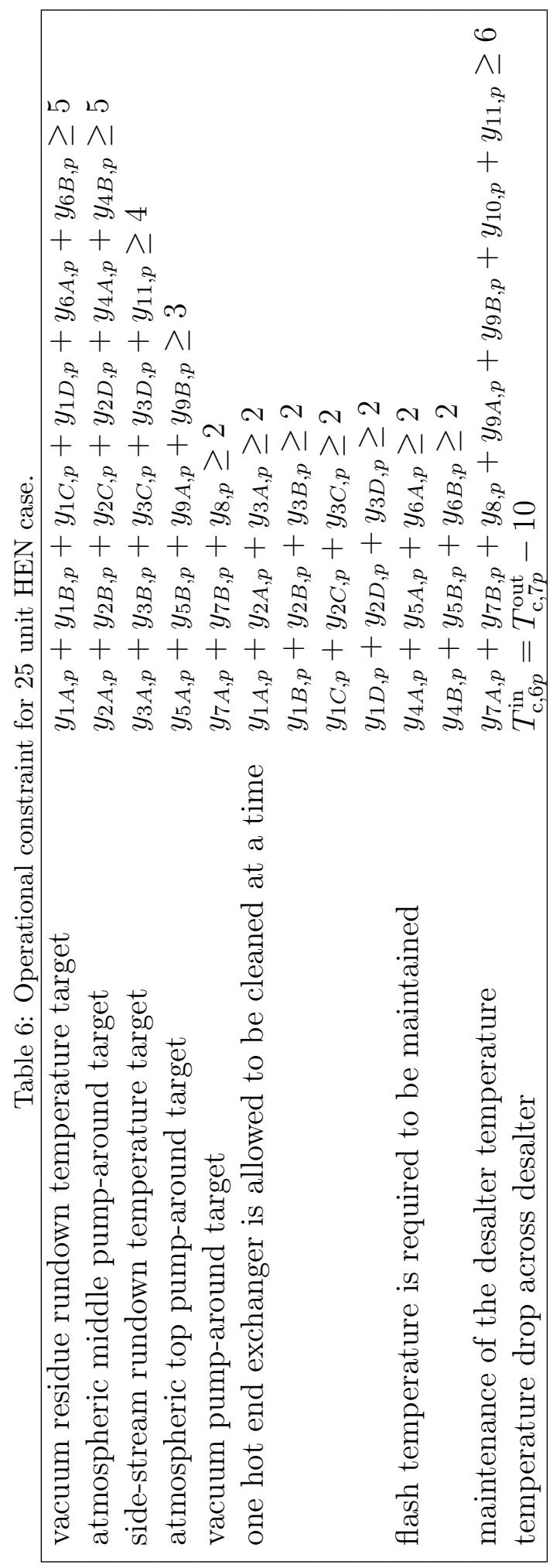


fouling, where there is an insignificant difference in savings. This is because of the existence of multiple local optima. It is noteworthy that Lavaja and Bagajewicz's (2004) MILP model is solved to global optimality whereas our model, being a non-convex MINLP model, is not. Despite this, we still obtain similar results.

For the 10 unit HEN (tables 14 and 15), although a general relation is seen in Lavaja and Bagajewicz's schedule where cleaning actions increase in the asymptotic fouling case vs. the linear one (from 10 to 11 cleanings), this drops down by 4 cleaning actions in our model as shown in tables 14 and 15. Only the last 3 units are cleaned here whilst there is a more distributed cleaning of units in the schedule of Lavaja and Bagajewicz, with half the units in the network undergoing cleaning during the operational horizon. Consequently, the cost of their schedule is slightly less than ours (£484k versus £493k as shown in table 9 ). This is a small difference of just over $1.5 \%$ in savings.

For all reported schedules there is an absence of cleaning actions near the start and the end of the operating horizon as there is little incentive to clean a relatively clean unit and there is little time for the cost of cleaning to be recovered towards the end of the operating horizon. If one were to increase the cost of cleaning further, this would limit the number of cleaning actions even more and increase the objective further. This can be used to determine which cleaning actions and/or exchangers are more important. For the 10 unit HEN, from tables 14 and 15, it can be seen that exchangers 9 and 10 are cleaned most frequently, indicating that these exchangers are more important in the network, while exchangers 1 and 2 in the linear and asymptotic models are not cleaned at all. Exchangers 9 and 10 are cleaned more often as they have the highest fouling rates as shown in table 3 . The fouling rate is not the only criterion that determines how often cleaning is done. For instance as shown in table 3 in Lavaja and Bagajewicz's (2004) schedule, despite the similar asymptotic fouling rates of exchangers 5 and 7 , the former is not cleaned at all while the latter is cleaned twice during the operating horizon. This is due to network sensitivity.

An important point to note is the bang-bang nature of these problems. The solutions 
of the relaxed models are completely integer i.e. a bang-bang control solution. Thus, the proposed rounding up scheme was not performed here. A number of schedules with similar objective values but different order of cleaning actions are obtained where very few fractional binary variables occur. These solutions are termed bang-singular. For the majority of cases, the range of objective values obtained in the 50 runs is quite narrow as shown in table 17, where the objective values only vary from as little as $£ 3 \mathrm{k}$ up to $£ 15 \mathrm{k}$ in the first 5 case studies. For the 10 unit asymptotic HEN case study, this range widens up to to £42k with a minimum of $£ 493 \mathrm{k}$ to a maximum of $£ 535 \mathrm{k}$, and up to $£ 28 \mathrm{k}$ for the 25 unit HEN case study with a variation of $£ 902 \mathrm{k}$ to $£ 930 \mathrm{k}$. Hence, for less complex networks and/or fouling models many runs at different starting points are not required to obtain a good solution.

A cost comparison only makes sense in the case studies appearing in Lavaja and Bagajewicz (2004) where the objective value for the no cleaning scenarios are similar (see tables 7 to 9). For the 25 unit HEN case studies, Smaili et al. (2002a) reported a lower objective associated with the no cleaning scenario representing $<11 \%$ difference (see table 10). This is partly attributed to our model retaining the fouling expressions in their dynamic form, which is more accurate. Smaïli et al. (2002a) discretised the system equations and thus assumed that variables such as temperature of hot and cold stream are fixed within each sub-period which is not a good approximation for large complex networks with extensive feedback of hot/cold streams. Temperatures in our model are interpreted continuously over time. The difference in the objective for the no cleaning scenario in the 25 unit HEN is also attributed to the different numerical methods used to the solve the equation sets.

For the 25 unit HEN case study our solution yields a saving of $36.2 \%$ with an overall cost of $£ 902 \mathrm{k}$, whereas the best reported cost produced by Smaïli et al. (2002a) using their BTA algorithm is £917k. Smaili et al. (2002a) were unable to generate a solution using the OA method. Our schedules have a small number of cleaning actions, in common with that of Smaili et al.. As in the 4 units over 18 months case study, no pattern is evident in the cleaning actions for the Smaïli et al. (2002a) method. More cleaning actions are performed 
in our schedule (37 versus 34 , table 16). Some features in common are that most exchangers are cleaned the same number of times as our schedule and certain exchangers are not cleaned at all (e.g. exchanger 10).

In terms of the distribution of the objective values for the 50 runs performed in each case, the results for each of the cases is narrowly dispersed around its associated mean value. The relative standard deviation (RSD) of the local optima for each of the cases considered lies in a narrow range of 0.8 to $1.5 \%$ (table 17). Furthermore, the difference between the maximum and minimum cost value is only £3k for the 4 unit heat exchanger case over a 12 month operating horizon, whereas this difference is the highest for the 10 unit HEN case subject to asymptotic fouling, at $£ 42 \mathrm{k}$. For the 10 unit HEN case subject to asymptotic fouling, the worst run results in a saving of $3.6 \%$ compared to $11.2 \%$ for the best solution achieved, while for the 4 unit heat exchanger case over a 12 month length of operation this is a saving of $19.3 \%$ in the worst case compared to $21.5 \%$ in the best case scenario.

The resource usage varies depending on fouling type, method used and problem size. Reasonable time for convergence is achieved for cases studies appearing in Lavaja and Bagajewicz (2004) and resource usage is practical even for the worst case: the 10 unit HEN with asymptotic fouling model required $942 \mathrm{CPU}$ s (15.7 CPU min), with the corresponding best case for this model being a modest 91 CPU s. Lavaja and Bagajewicz (2004) stated that the time to solve the 10 unit HEN case was impractical, therefore in addition to reformulating their model into a MILP problem they used a decomposition procedure to decrease the computational time. They also stated that they kept the linearity of the expressions with the aim of having better chances of capturing the global optimum. From our findings, neither of these are required. In comparison, the resource usage becomes expensive for the 25 unit HEN case study. This required 55,243 CPU s (15.3 CPU hr) with 38,603 function evaluations in the worst case. This is due to the implementation approach whereby gradients are estimated using finite differences in the MATLAB ${ }^{\circledR}$ optimiser.

The computational cost is proportional to the number of finite difference calculations 
required, with each finite difference calculation requiring a full dynamic system simulation; for larger problems, this leads to a significant computational cost. For example, for the single heat exchanger case under linear fouling for an operating horizon of 24 periods, an average of 11 gradient calculations is required with each one requiring 24 finite difference calculations, as shown in Table 17. This accounts for the average computational cost of $30 \mathrm{CPU}$ s. In the case of the 25 unit heat exchanger network under linear fouling over 36 periods results in a much larger average computational time of 39,611 CPU s (11 CPU hr). In this case, there is an average of 31 gradient calculations each of them requiring 900 finite difference calculations.

Future applications of the multistage optimal control approach will include the reduction of CPU time such that it becomes significantly smaller in larger and more complex networks. This will be achieved through gradient estimation using sensitivity equations. Furthermore, future work will involve extending the range of case studies in HENs to include pressure drop constraints, variable throughput, and optimisation of operating conditions such as the consumption of utilities. This approach is not limited to HENs, and future work will focus on the optimisation of general scheduling maintenance problems.

\section{Critique}

This work has demonstrated that the heat exchanger cleaning scheduling problem as posed, considering all potential cleaning actions, can be solved for large networks and larger numbers of actions than previously achieved through the recognition of the task as an optimal control problem where the solutions fit bang-bang characteristics. We here review which aspects of the scheduling problem which may be encountered in practice have been included in the work, and those which have not, in order to identify the scope and potential for further development.

Aspects which have been included are the distribution of heat duties within networks in response to cleaning actions, and their evolution; linear and nonlinear (asymptotic) fouling 


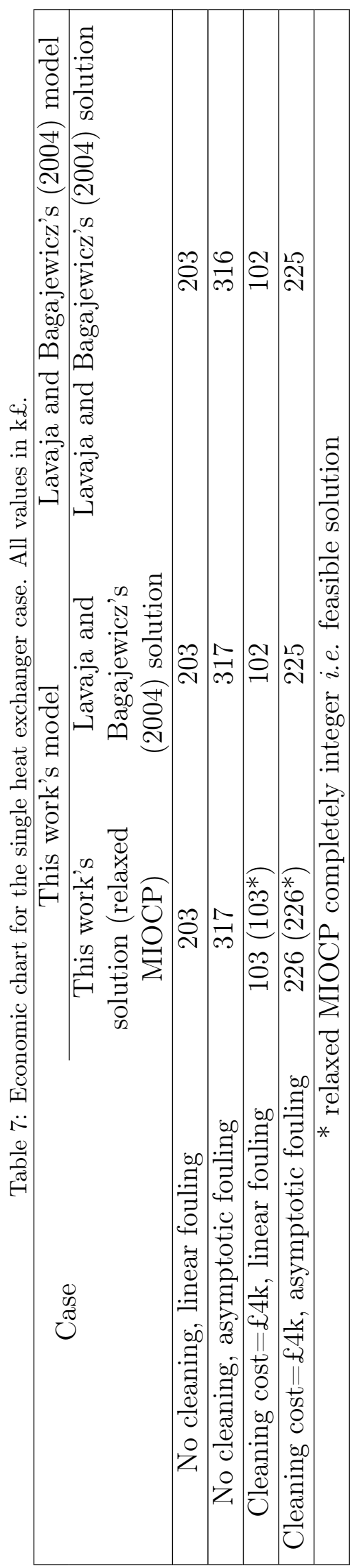




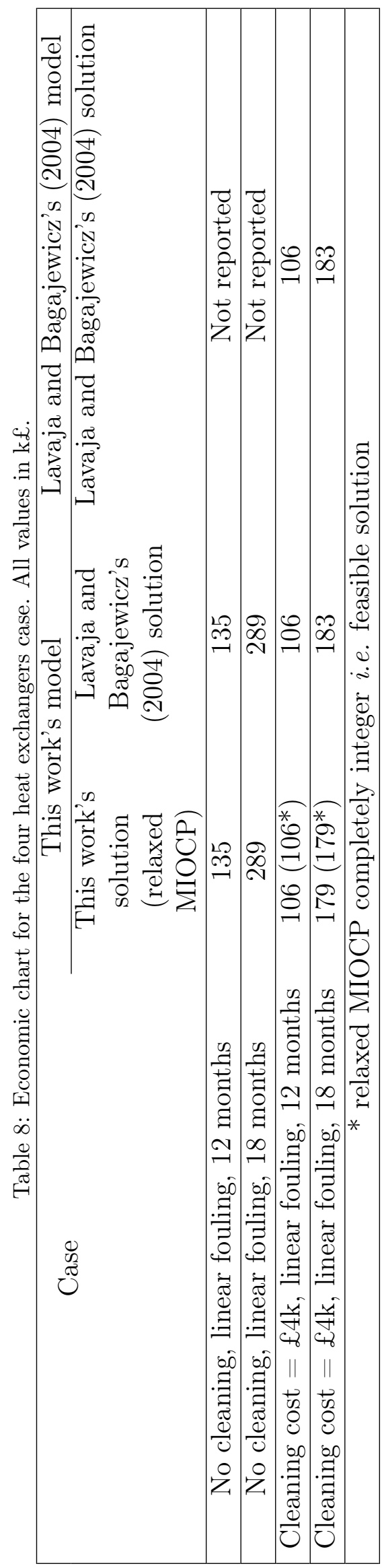




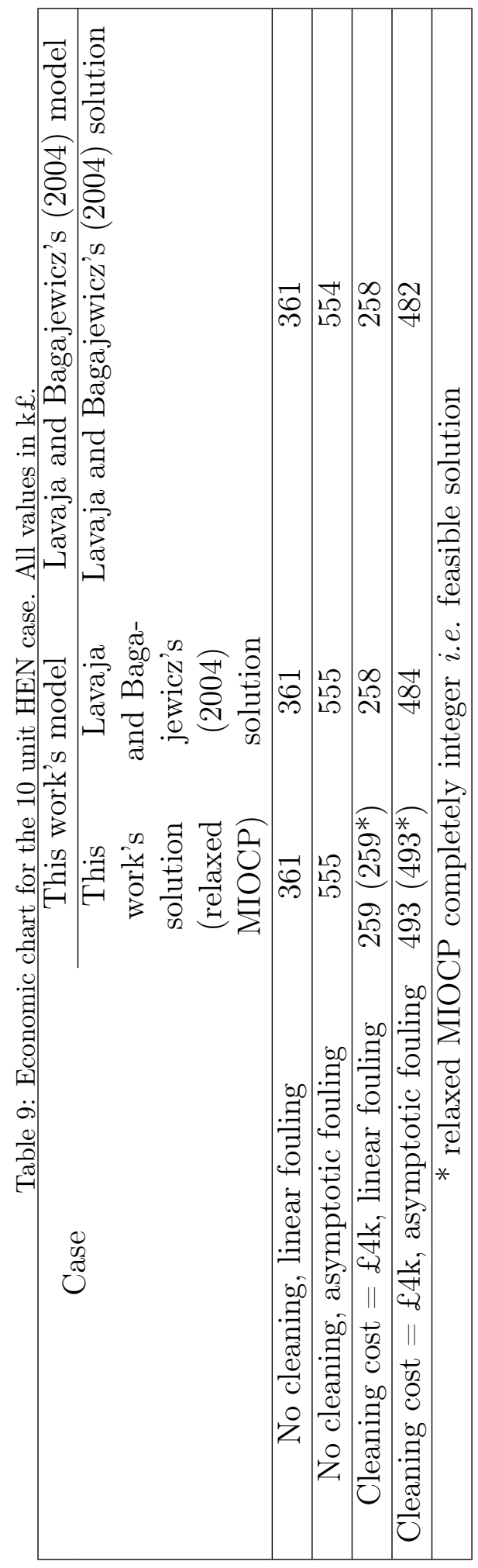




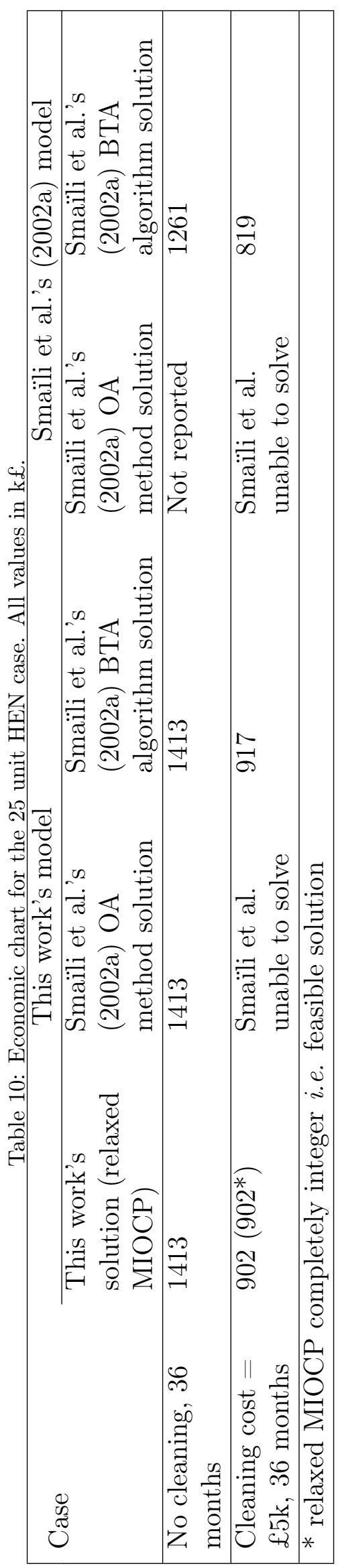




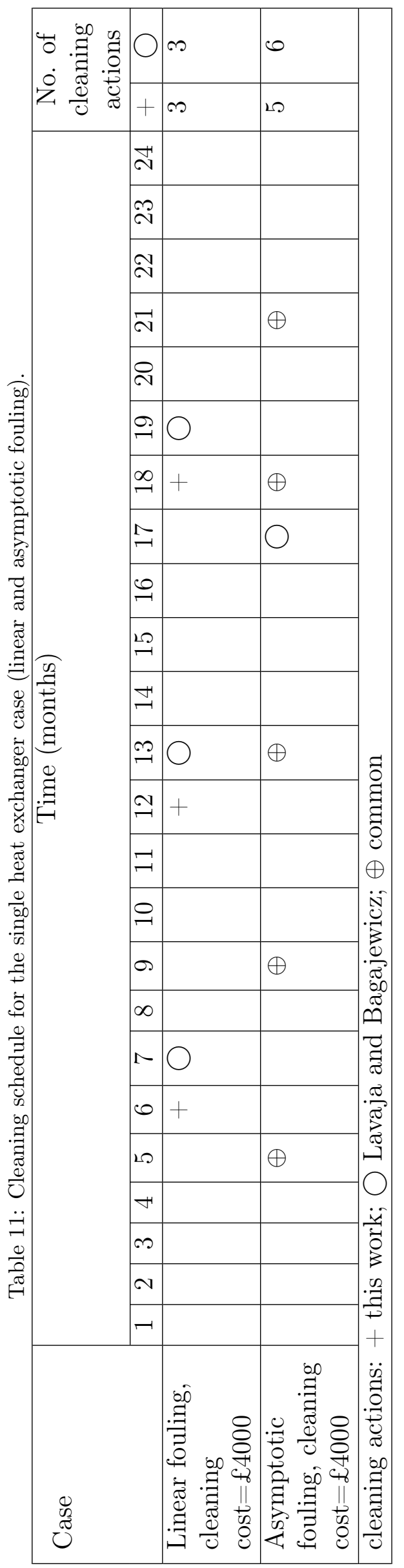




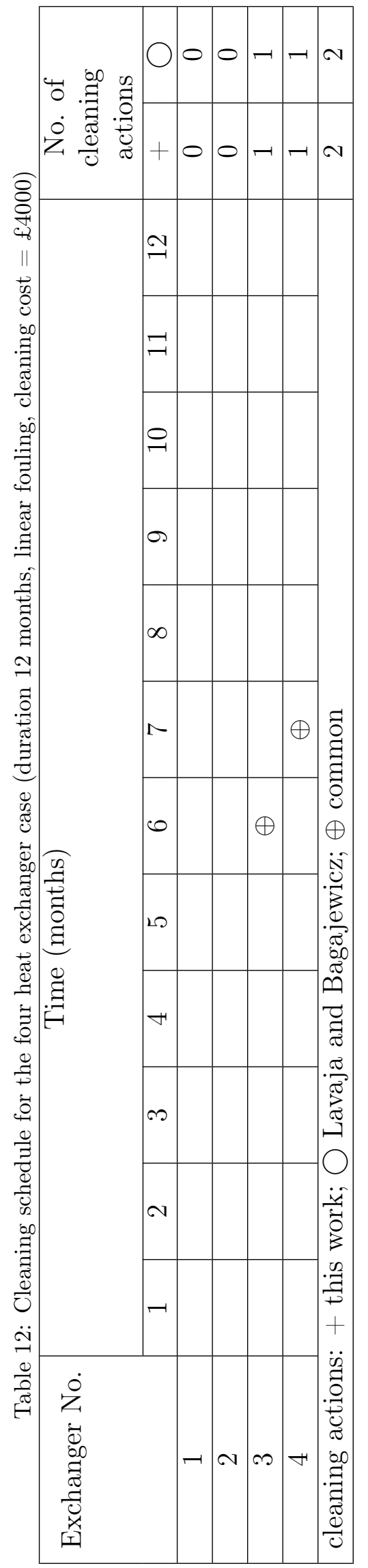




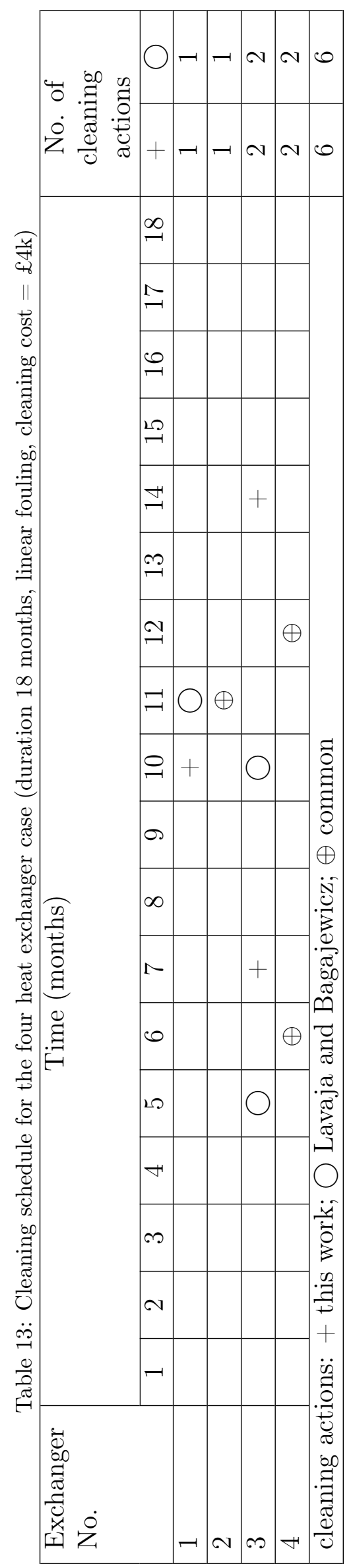




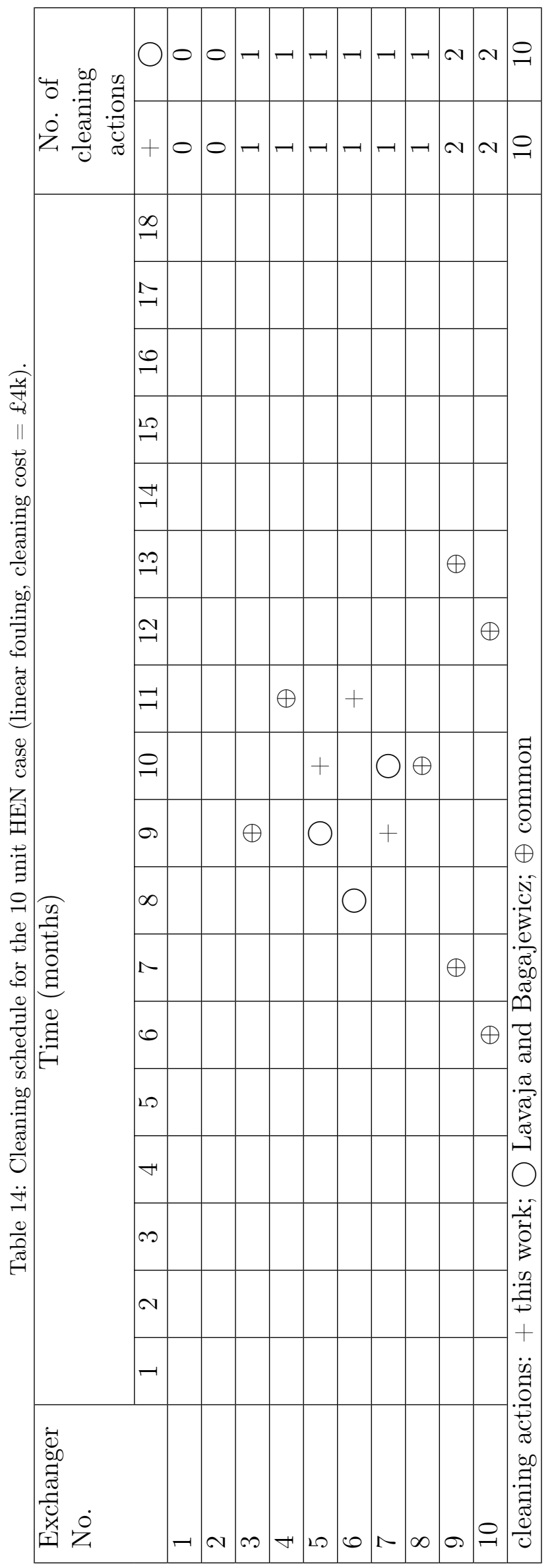




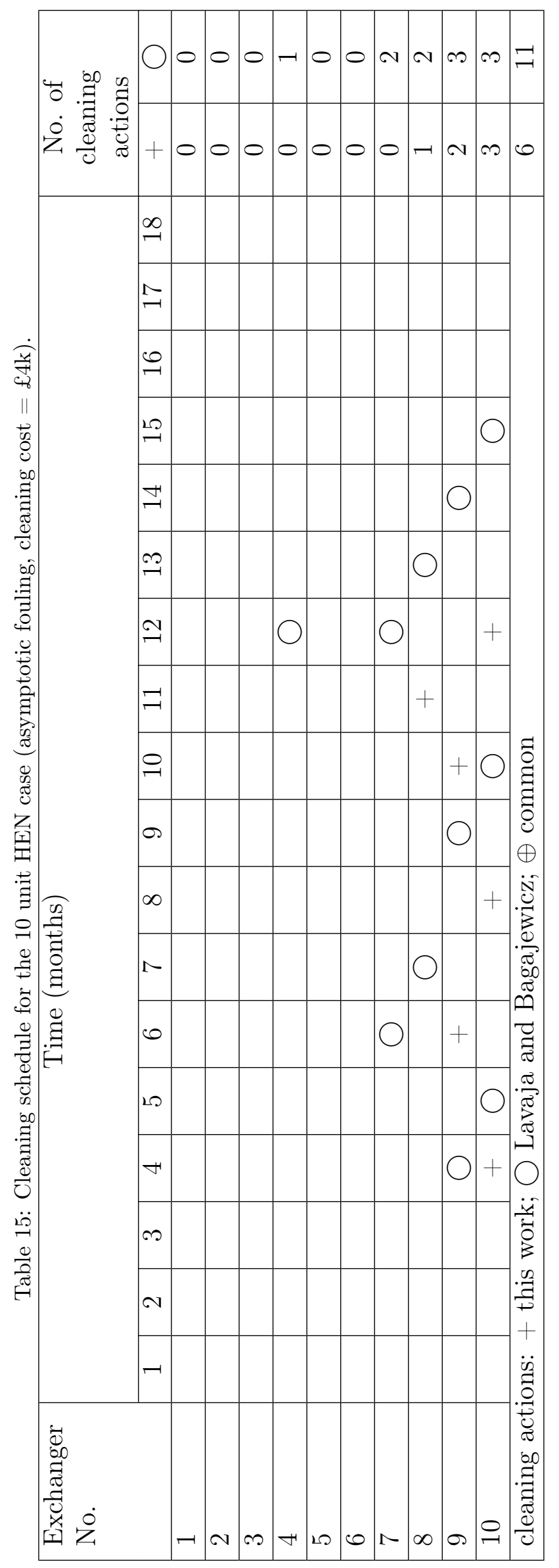




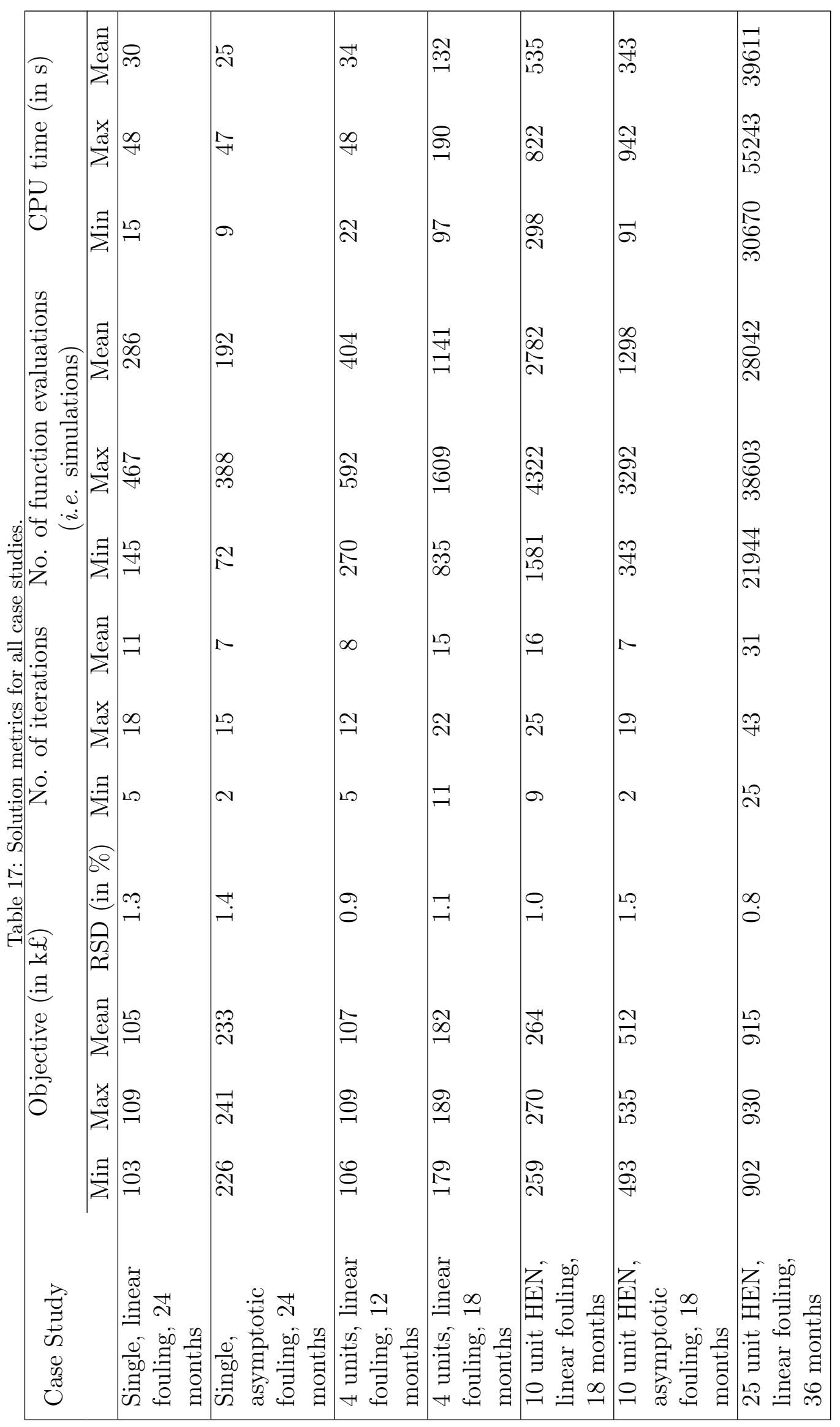


behaviour; and constraints on the selection of combination of cleaning actions representing pump-around targets, rundown temperature targets, flash temperature maintenance, etc. Aspects presented by other workers which could be included without loss of generality, but requiring more detailed modelling and therefore solution time, include the choice between two cleaning actions (Pogiatzis et al., 2011) and temperature target constraints (e.g. desalter temperature, see Ishiyama et al. (2010)).

Those not included can be grouped as follows:

(i) Nonlinearity arising from fouling phenomena. Fouling rates are known to depend strongly on temperature, and will therefore vary in an exchanger over time as fouling changes the temperature distribution within a network. This level of detailed modelling can be incorporated in greedy (Ishiyama et al., 2009a) and genetic algorithm approaches (Rodriguez and Smith, 2007), at the expense of ensuring global optimality, as well as in these total horizon approaches.

(ii) Nonlinearity arising from network dynamics. Fouling deposits change the pressure drop across a heat exchanger as well as its heat transfer performance. The network model presented here assumes constant stream flow rates, but fouling in practice can give rise to flow redistribution between parallel streams as well as throughput reduction as a result of pumping limitations (Yeap et al., 2004; Ishiyama et al., 2008). Changes in flow rate affect both local fouling rates and the objective function, and network models incorporating pressure drop and throughput dynamics have been constructed. The relationship between fouling resistance, pressure drop and throughput is not linear: depending on the network configuration, it can feature a threshold followed by a quasi-parabolic region. The heat duty in the objective function (equation $(24)$ ) then contains a product of two variables ( $\dot{F}_{c}$ and CIT), and with an appropriate formulation, this is amenable to this total horizon approach.

(iii) Uncertainty in fouling models and model parameters. Wilson et al. (2017) recently reviewed the progress in quantitative fouling models for crude oil fouling. They reported three areas where systematic uncertainty arise in models for predicting the fouling rates in 
crude oil as related to the problems presented here:

(a) The fouling models are semi-empirical and the relationship to crude oil composition and characteristics has yet to be established, so one cannot predict, for example, whether linear or asymptotic fouling will be observed in a given unit.

(b) Fouling rates for complex fluids such as crude oil are rarely studied under controlled conditions. In practice many operators used fouling models constructed from reconciliation and interpretation of plant fouling data. These are subject to uncertainties in measurement and calculation, so the accuracy of the fouling rate data is low.

(c) The relationship between fouling rates and crude composition is unknown. In most applications the crude being processed varies with time so the rate(s) will also vary. This is one of the reasons why plant fouling data, used to quantify fouling model parameters, contain noticeable scatter and variation. These areas mean that, in practice, scheduling calculations must be able to consider a range of likely fouling rates.

There is a conflict between aspects (i) and (ii), and (iii): the increased model complexity in the former means that multiple condition testing, as required by (iii), will require considerable resource. The desire to account for known, deterministic phenomena must be balanced against the limitations to tractability introduced by those phenomena. From an engineering perspective, the question to be asked is which essential features of the problem must be included, at a suitable level of detail, to achieve the desired outcome.

Aspects (i) and (ii) will require special reformulation to be incorporated in a suitable level of detail for some practical cases with total horizon approaches, such as the one described in this work. These approaches are, however, ideally suited for combination with algorithms for designing heat exchanger networks as they can generate estimates for expecting optimal operating performance, including considerations of uncertainty in fouling (and operating parameters).

For the case of a crude preheat train, the initial network design would yield temperature and flow rate conditions for which fouling rates could be estimated. The operation of this 
network, with cleaning schedules calculated for a portfolio of fouling rates, could then be quantified (and key exchangers identified for design attention), and this information used to update the design. Wang and Smith (2013) employed simulated annealing approaches to identify fouling resistant preheat train designs but did not incorporate cleaning aspects in their consideration of network performance: the current work now makes this a tractable problem and one worthy of attention. Current network complexities may prohibit application of a full optimisation based methodology for the scheduling of cleaning, and hence currently the preference in industry is to use heuristic or greedy approaches. However, the contribution of this work is to show that optimisation based methodologies can be general enough to encapsulate both complexity and different operating modes and this will be explored further in future work.

\section{Conclusions}

An alternative methodology to the solution of the HEN cleaning scheduling problem is presented here by recognising, for the first time, that this optimisation model is in actuality a MIOCP which exhibits bang-bang behaviour. This proves to be an efficient and robust approach and has been compared with 3 different methods: a direct MINLP approach (OA), reformulation of the MINLP to an MILP model, and a stochastic optimisation technique (BTA algorithm).

The multistage optimal control formulation using the feasible path approach does not suffer from failures in convergence and is thus reliable, contrary to the OA method which fails to produce a solution in larger and more complex networks. The feasible path approach as implemented is shown to be very competitive. Optimal solutions reported here are all bang-bang in the controls. As a result, these particular case studies did not require any heuristic approaches to be applied. In comparison to the classical methods, economic values are similar and in some instances better than those obtained. The cleaning schedules showed several conventional characteristics, with key exchangers being cleaned more often. However, 
the allocation of cleaning actions was often not systematic, i.e. unpredictable.

\section{Acknowledgements}

Support of this research by the Ministry of Higher Education in the Sultanate of Oman and Petroleum Development Oman (PDO) is gratefully acknowledged.

\section{References}

Belghith, S. F., Lamnabhi-Lagarrigue, F., Rosset, M. M., 1986. Algebraic and geometric methods in nonlinear control theory. Vol. 29. Springer Netherlands, Dordrecht.

Bellman, R., Glicksberg, I., Gross, O., 1956. On the bang-bang control problem. Quaterly of Applied Mathematics 14 (1), 11-18.

Blakemore, N., Aris, R., 1962. Studies in optimization-V. The bang-bang control of a batch reactor. Chemical Engineering Science 17, 591-598.

Bryson, A. E., Ho, Y.-C., 1975. Applied optimal control: Optimization, estimation, and control. Hemisphere Publishing Corporation, New York-Washington-Philadelphia-London.

Casado, E., 1990. Model optimizes exchanger cleaning. Hydrocarbon Processing 69 (8), 7176.

Fouskakis, D., Draper, D., 2002. Stochastic optimization: a review. International Statistical Review 70 (3), 315-349.

Georgiadis, M. C., Papageorgiou, L. G., 2000. Optimal energy and cleaning management in heat exchanger networks under fouling. Chemical Engineering Research and Design 78 (2), 168-179.

Gonçalves, C. D. O., Queiroz, E. M., Pessoa, F. L. P., Liporace, F. S., Oliveira, S. G., Costa, A. L. H., 2014. Heuristic optimization of the cleaning schedule of crude preheat trains. Applied Thermal Engineering 73 (1), 1-12. 
Ishiyama, E. M., Heins, A. V., Paterson, W. R., Spinelli, L., Wilson, D. I., 2010. Scheduling cleaning in a crude oil preheat train subject to fouling: Incorporating desalter control. Applied Thermal Engineering 30 (13), 1852-1862.

Ishiyama, E. M., Paterson, W. R., Wilson, D. I., 2008. Thermo-hydraulic channelling in parallel heat exchangers subject to fouling. Chemical Engineering Science 63 (13), 34003410.

Ishiyama, E. M., Paterson, W. R., Wilson, D. I., 2009a. Platform for techno-economic analysis of fouling mitigation options in refinery preheat trains. Energy and Fuels 23 (3), 1323-1337.

Ishiyama, E. M., Paterson, W. R., Wilson, D. I., 2009b. The effect of fouling on heat transfer, pressure drop, and throughput in refinery preheat trains: optimization of cleaning schedules. Heat Transfer Engineering 30 (10-11), 805-814.

Ishiyama, E. M., Paterson, W. R., Wilson, D. I., 2011. Optimum cleaning cycles for heat transfer equipment undergoing fouling and ageing. Chemical Engineering Science 66 (4), 604-612.

Lavaja, J. H., Bagajewicz, M. J., 2004. On a new MILP model for the planning of heatexchanger network cleaning. Industrial \& Engineering Chemistry Research 43 (14), 39243938.

Mohler, R., 1973. Bilinear control processes: with applications to engineering, ecology, and medicine. Academic Press, New York.

Pogiatzis, T., Vassiliadis, V. S., Wilson, D. I., 2011. An MINLP formulation for scheduling the cleaning of heat exchanger networks subject to fouling and ageing. In: Malayeri, M. R., Müller-Steinhagen, H., Watkinson, A. P. (Eds.), Preceedings of International Conference on Heat Exchanger Fouling and Cleaning. Vol. 2011. Crete Island, pp. 349-356. 
Pogiatzis, T., Wilson, D. I., Vassiliadis, V., 2012. Scheduling the cleaning actions for a fouled heat exchanger subject to ageing: MINLP formulation. Computers \& Chemical Engineering $39,179-185$.

Pontryagin, L. S., Boltyanskii, V. G., Gamkrelidze, R. V., Mishchenko, E. F., 1962. The mathematical theory of optimal processes. Wiley-Interscience, New York.

Process Systems Enterprise, 2017. gPROMS, www.psentersprise.com/gproms.

Pugh, S. J., Hewitt, G. F., Müller-Steinhagen, H., 2001. Heat exchanger fouling in the pre-heat train of a crude oil distillation unit - The development of a "user guide". In: Proceedings of the 4th International Conference on Heat Exchanger Fouling, Fundamental Approaches \& Technical Solutions. No. July. Davos.

Rodriguez, C., Smith, R., 2007. Optimization of operating conditions for mitigating fouling in heat exchanger networks. Chemical Engineering Research and Design 85 (6), 839-851.

Sager, S., 2009. Reformulations and algorithms for the optimization of switching decisions in nonlinear optimal control. Journal of Process Control 19 (8), 1238-1247.

Smaïli, F., Angadi, D. K., Hatch, C. M., Herbert, O., Vassiliadis, V. S., Wilson, D. I., 1999. Optimization of scheduling of cleaning in heat exchanger networks subject to fouling. Food and Bioproducts Processing 77 (2), 159-164.

Smaïli, F., Vassiliadis, V. S., Wilson, D. I., 2001. Mitigation of fouling in refinery heat exchanger networks by optimal management of cleaning. Energy \& Fuels 15 (5), 10381056.

Smaïli, F., Vassiliadis, V. S., Wilson, D. I., 2002a. Long-term scheduling of cleaning of heat exchanger networks. Chemical Engineering Research and Design 80 (6), 561-578.

Smaïli, F., Vassiliadis, V. S., Wilson, D. I., 2002b. Optimization of cleaning schedules in heat 
exchanger networks subject to fouling. Chemical Engineering Communications 189 (11), $1517-1549$.

Taborek, J., Aoki, T., Ritter, R. B., Palen, J. W., 1972. Fouling: The major unresolved problem in heat transfer.

The MathWorks Inc., 2016. MATLAB and Optimisation Toolbox.

Vassiliadis, V. S., 1993. Computational solution of dynamic optimization problems with general differential-algebraic constraints. Ph.D. thesis, University of London.

Wang, Y., Smith, R., 2013. Retrofit of a heat-exchanger network by considering heat-transfer enhancement and fouling. Industrial and Engineering Chemistry Research 52 (25), 85278537.

Watkinson, A. P., 1988. Critical review of organic fluid fouling. Argonne national laboratory report No. ANL/CNSV-TM-208. Tech. rep.

Wilson, D. I., Ishiyama, E. M., Polley, G. T., 2017. Twenty years of Ebert and Panchal what next? Heat Transfer Engineering 38 (7-8), 669-680.

Yeap, B., Wilson, D., Polley, G., Pugh, S., 2004. Mitigation of crude oil refinery heat exchanger fouling through retrofits based on thermo-hydraulic fouling models. Chemical Engineering Research and Design $82(1), 53-71$.

Zandvliet, M., Bosgra, O., Jansen, J., Van den Hof, P., Kraaijevanger, J., 2007. Bangbang control and singular arcs in reservoir flooding. Journal of Petroleum Science and Engineering 58 (1-2), 186-200. 